\title{
Modeling, analyzing and simulating the dynamics of Lassa fever in Nigeria
}

\author{
Mayowa M. Ojo ${ }^{1,2^{*}}$ (D) and Emile Franc Doungmo Goufo ${ }^{1}$
}

\author{
*Correspondence: \\ mmojomth@gmail.com \\ ${ }^{2}$ Thermo Fisher Scientific, \\ Microbiology Division, \\ Lenexa, USA \\ Full list of author information \\ is available at the end of the \\ article
}

\begin{abstract}
Lassa fever is an infectious and zoonotic disease with incidence ranging between a hundred to three hundred thousand cases, with approximately five thousand deaths reported yearly in West Africa. This disease has become endemic in the Lassa belt of Sub-Saharan Africa, thus increasing the health burden in these regions including Nigeria. A deterministic mathematical model is presented to study the dynamics of Lassa fever in Nigeria. The model describes the transmission between two interacting hosts, namely the human and rodent populations. Using the cumulative number of cases reported by the Nigerian Centre for Disease Control within the first week of January 2020 through the eleventh week in 2021, we performed the model fitting and parameterization using the nonlinear least square method. The reproduction number $\mathcal{R}_{0}$ which measures the potential spread of Lassa fever in the population is used to investigate the local and global stability of the system. The result shows that the model system is locally and globally asymptomatically stable whenever $\mathcal{R}_{0}<1$, otherwise it is unstable. Furthermore, the endemic equilibrium stability is investigated and the criteria for the existence of the phenomenon of bifurcation is presented. We performed the sensitivity analysis of each reproduction number parameter and solutions of the developed model are derived through an iterative numerical technique, a six-stage fifth-order Runge-Kutta method. Numerical simulations of the total infected human population $\left(E_{h}+I_{h}\right)$ under different numerical values (controlled parameters) are presented. The result from this study shows that combined controlled parameters made the total infected human population decline faster and thus reduces Lassa fever's burden on the population.
\end{abstract}

Keywords: Stability analysis, Sensitivity analysis, Model fitting, Controlled parameter, Reproduction number, Lassa fever

\section{Introduction}

Lassa fever is an infectious disease and a zoonotic viral illness which is also called Lassa hemorrhagic fever. It is instigated by the Lassa virus, a single-stranded RNA virus from the Arenaviridae family $[1,2]$. The main host of this virus is the mastomys natalensis, also known as a multimammate rat. It is known in Sub-Saharan African as one of the most common rodent species [3-5]. Although Lassa fever (LF) was first described in the 1950s, the viral particle responsible for its cause was first identified in 1969 in the northern region of Nigeria. This disease was named after Lassa, a town in Borno state

(c) The Author(s) 2022. Open Access This article is licensed under a Creative Commons Attribution 4.0 International License, which permits use, sharing, adaptation, distribution and reproduction in any medium or format, as long as you give appropriate credit to the original author(s) and the source, provide a link to the Creative Commons licence, and indicate if changes were made. The images or other third party material in this article are included in the article's Creative Commons licence, unless indicated otherwise in a credit line to the material. If material is not included in the article's Creative Commons licence and your intended use is not permitted by statutory regulation or exceeds the permitted use, you will need to obtain permission directly from the copyright holder. To view a copy of this licence, visit http:// creativecommons.org/licenses/by/4.0/. 
Nigeria where it was first identified. However, it has become endemic and a health challenge in Western African. According to the Centers for Disease Control and Prevention (CDC) and World Health Organization (WHO), the yearly estimated incidence in West Africa ranges from a hundred to three hundred thousand cases with nearly five thousand deaths [6-8]. The eastern and western regions of West Africa have been identified as the high-risk area for Lassa fever with the regular widespread outbreak from the Lassa belt. The countries in the Lassa belt include Liberia, Guinea, Sierra Leone, and Nigeria [6, 9-11]. Many outbreaks have been reported from these regions over the years, among these is the largest epidemic reported in Nigeria, the country we take as a case study in this work. In 2018, Nigeria recorded an outbreak of Lassa fever which swept through eighteen out of the thirty-six states of the country. Over 400 confirmed cases were reported, and this was recorded as the largest outbreak [12]. However, following this incidence, Lassa fever cases have been increasing with an upsurge in both confirmed cases and deaths. Using the reported cases obtained through Nigeria Centre for Disease Control (NCDC) database [13], we depict the trend of confirmed cases and deaths from 2018 to 2020 for Nigeria in Fig. 1. Although the prevalence of this disease is associated with an increase in the host reservoir, which is mainly driven by the ecological climate factor rainfall, various factors such as insufficient health facilities, polluted environment, and poor personal hygiene have contributed largely to the increase of cases yearly. Since

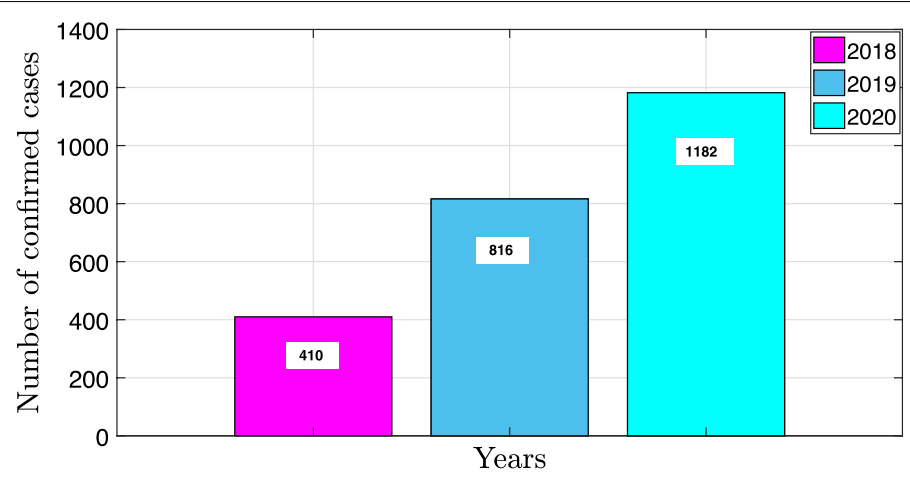

(a)

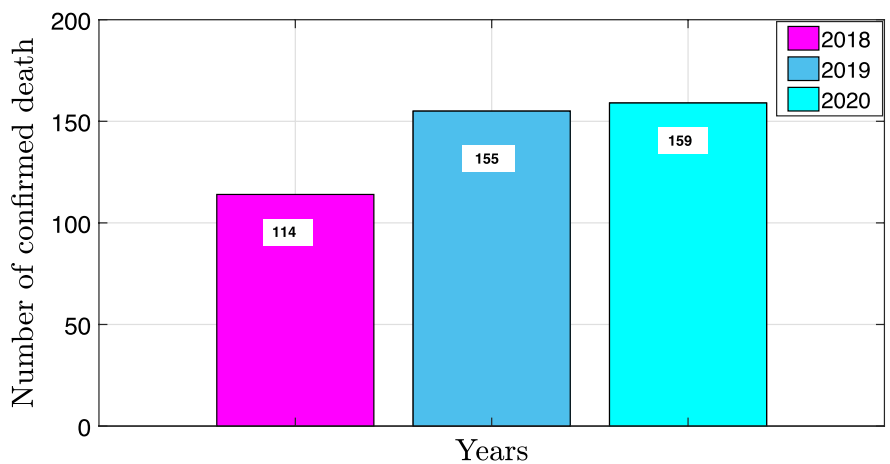

(b)

Fig. 1 Number of reported cases a Confirmed cases; $\mathbf{b}$ Confirmed death. Reported cases are obtained from NCDC database [13], from 2018 to 2020 
the mastomys rodents migrate from their natural habitation to the human environment during the rainy season, a decrease in the prevalence of Lassa fever is dependent on human efforts in reducing the transmission probability of this disease [3, 14].

The Lassa virus is primarily spread to humans through human contact with food or substances that are contaminated by the urine or feces of an infected rodent [9], while secondary infection from human-to-human and laboratory transmissions are likewise possible [10,15]. Lassa fever has an incubation period between 6 and 21 days, hence, following this exposure period, infected humans are expected to start showing symptoms of the disease. Although about eighty percent of infected humans have only slight symptoms such as headaches, cough, muscle pain, sore throat, weakness, and fever. However, in severe cases, an infected human can develop more complications such as facial swelling, bleeding from the nose, respiratory distress, and low blood pressure $[2,11,16]$. In a more critical situation, this disease can lead to death within fourteen days after the first appearance of the symptoms, due to neurological problems $[2,16]$.

Due to the absence of a vaccine against Lassa fever, prevention against infection has an important role in controlling the transmission of this disease in the population. Currently, since the eradication of mastomys rodent population is unrealistic, the present ways of avoiding the spread of this infection include the facilitation of good personal hygiene to avoid contact with infected rodents' secretions or excretions, and implementation of standard health facilities for effective testing, diagnosing and treatment of patients [10]. In addition, ribavirin is an antiviral drug that has been declared as an effective treatment for Lassa fever patients, if administered at the premature period of the infection $[7,9]$.

Over the decade, mathematical models have become vital tools in studying the dynamics of diseases in a given population. The recent development of the use of mathematical models has covered many fields, using different methods. Among them includes the use of fractional calculus on modeling and analysis of applied problems which includes infectious diseases field. For examples of these recent studies, see [17-21] and the references therein. Many models such as [2, 22-33], has been developed for numerous diseases, to answer specific questions in an attempt to contribute to the understanding of the epidemiology of such disease under study. More specifically, studies have been carried out to further provide information on the transmission dynamics of Lassa fever (see [3, 7-9, 14, 16, 34-36]). A few researchers have investigated the transmission dynamics of Lassa fever in Nigeria, using a different mathematical modeling approach. Among them is the work of Salihu [9]. The authors employed a mechanistic modeling approach to study the large-scale Lassa fever epidemics in Nigeria from the year 2016 to 2019. To understand the transmission dynamics of Lassa fever epidemics in Nigeria, the model describes the interaction among rodent and human populations by integrating isolation, quarantine, and also hospitalization compartment. Their results suggest that an increase in quarantine and isolation of infected people will decrease the transmission of Lassa fever from human to human. In [3], Zhao studied the large-scale of Lassa fever outbreak in different parts of Nigeria. The authors investigated some epidemiological features of the epidemic by measuring the correlation between the reproduction number of the disease and local rainfall, using the three-parameter logistic, Richards growth model, Gompertz, and Weibull growth model. They further fit the respective growth models to the surveillance data to evaluate the reproduction number with the 
respective epidemic turning points. The results from this study show that rainfall has an enormous influence on the transmission of Lassa fever in Nigeria.

In this study, we present a six compartmental deterministic model, using a system of ordinary differential equations to gain insight into the transmission dynamics of Lassa fever within humans and rodents population. One of the uniqueness of this work is that we parameterize the model with the real data (reported cases from Nigeria), from the first week of January 2020 to the eleventh week of 2021. This will make model predictions more meaningful and, as a result, provide a better understanding of the dynamics of Lassa fever in Nigeria. The rest of the paper is structured as follows. The model formulation is presented in "Section Model formulation", while the basic properties of the model, such as positivity of solutions, and invariant region are presented in Section "Basic properties of the model". In "Section Mathematical analysis of the model", the existence and stability of the Lassa fever model are investigated. This includes local and global stability of the Lassa feverfree equilibrium, the existence, and stability of the endemic equilibria, and the condition for the existence of the bifurcation phenomenon. Parameter estimation and data fitting were carried out in "Section Parameter estimation and data fitting". This includes the sensitivity analysis of each reproduction number parameter. We present the numerical simulations, discussion, and conclusions of the results in "Section Results and discussion".

\section{Methods}

\section{Model formulation}

To achieve the main aim of this study, we develop, analyze, parameterize and simulate an epidemic model that describes the transmission dynamics of Lassa fever in Nigeria. Since the transmission of Lassa fever requires an interaction between two-interacting populations [6], we derived our model by classifying the host population into the human and rodent populations. Furthermore, according to human disease status, the total human population at continuous-time $t$ denoted by $N_{h}(t)$ is stratified into mutually-exclusive compartments. Precisely, the total human population $N_{h}(t)$ is grouped into the sub-populations of individuals who are susceptible $S_{h}(t)$, exposed $E_{h}(t)$, infectious $I_{h}(t)$, and recovered $R_{h}(t)$. Thus, the total human population $N_{h}(t)$ is given as

$$
N_{h}(t)=S_{h}(t)+E_{h}(t)+I_{h}(t)+R_{h}(t)
$$

Similarly, the total rodent population at continuous-time $t$ denoted by $N_{r}(t)$ is divided into two compartments, namely: susceptible rodents $S_{r}(t)$ and infectious rodents $I_{r}(t)$. Hence, the total rodent population $N_{r}(t)$ is given as

$$
N_{r}(t)=S_{r}(t)+I_{r}(t)
$$

The susceptible human population is generated through recruitment by birth or immigration at a rate $\pi_{h}$. In addition, since reinfection with Lassa virus is possible [5, 37], we assume that the susceptible populace is additionally increased by immunity loss of recovered individuals at a rate $\xi_{h}$. Since all living beings are subjected to death, all subpopulations are liable to be reduced by the natural death (death not due to the disease), hence the susceptible human population is depopulated by a natural death at the rate $\mu_{h}$. Furthermore, this population is reduced after infection with the Lassa fever virus due to effective contact with an infectious human or infectious rodent at the rate 


$$
\lambda_{h}=\frac{\beta_{r h} I_{r}}{N_{h}}+\frac{\beta_{h} I_{h}}{N_{h}}
$$

The parameter $\beta_{r h}$ represents the effective transmission probability from rodent-tohuman, which could be through direct contact with contaminated food by the urine or excretes of an infectious rodent, while $\beta_{h}$ represents the effective transmission probability from human-to-human through dust particles via the mucous membranes or skin breaks of human, or through sharing of medical equipment with infectious individuals without adequate sterilization [7,9]. Thus, the susceptible human population at any given time $t$ is

$$
\frac{\mathrm{d} S_{h}}{\mathrm{~d} t}=\pi_{h}+\xi_{h} R_{h}-\lambda_{h} S_{h}-\mu_{h} S_{h}
$$

The exposed human population is derived from an infection occurring from the susceptible population. This populace is reduced by natural death $\mu_{h}$ and the disease progression to the infectious population at the rate $\sigma_{h}$. It is imperative to note that, exposed individuals are infected with the Lassa fever virus but are not showing symptoms yet. Following the disease incubation period which is between $6-21$ days $[2,11]$, such individuals progress to infectious population. This is the stage whereby they start showing symptoms of the disease. Thus, the exposed human population at any time $t$ is given as

$$
\frac{\mathrm{d} E_{h}}{\mathrm{~d} t}=\lambda_{h} S_{h}-\left(\sigma_{h}+\mu_{h}\right) E_{h}
$$

The infectious human compartment is populated as a result of the progression rate from the exposed human population. The population is reduced by the recovery rate due to treatment at rate $\tau_{h}$, natural death $\mu_{h}$, and disease-induced death (death caused by Lassa fever) at the rate $\delta_{h}$. The infectious human population is given as

$$
\frac{\mathrm{d} I_{h}}{\mathrm{~d} t}=\sigma_{h} E_{h}-\left(\tau_{h}+\mu_{h}+\delta_{h}\right) I_{h}
$$

Following early treatment of individuals diagnosed of Lassa fever disease, such individuals recover and progress to increase the recovered human population. However, since recovered individuals can be re-infected of the disease [5, 37], the recovered human populace is reduced by loss of immunity at rate $\xi_{h}$ and natural death at the rate $\mu_{h}$. Hence, the recovered human population is given as

$$
\frac{\mathrm{d} R_{h}}{\mathrm{~d} t}=\tau_{h} I_{h}-\left(\mu_{h}+\xi_{h}\right) R_{h}
$$

The susceptible rodents population is generated by the recruitment of rodent through birth at a rate $\pi_{r}$. This sub-population is reduced by natural death with the rate $\mu_{r}$, and is further decreased following infection with Lassa virus due to effective contact with an infectious human or rodent at the rate

$$
\lambda_{r}=\frac{\beta_{h r} I_{h}}{N_{h}}+\frac{\beta_{r} I_{r}}{N_{r}}
$$


The parameters $\beta_{h r}$ represents the effective transmission probability from human-torodent, while $\beta_{r}$ represents the effective transmission probability from rodent-to-rodent. Thus, the susceptible rodent population at any time $t$ is given as

$$
\frac{\mathrm{d} S_{r}}{\mathrm{~d} t}=\pi_{r}-\lambda_{r} S_{r}-\mu_{r} S_{r}
$$

The infectious rodent population is derived from infection occurring from the susceptible rodent population, while depopulated by natural death of rodents at rate $\mu_{r}$. Thus, the infectious rodent population is given as

$$
\frac{\mathrm{d} I_{r}}{\mathrm{~d} t}=\lambda_{r} S_{r}-\mu_{r} I_{r}
$$

Hence, based on the overall process explained above, we present below a six compartmental deterministic systems of nonlinear ordinary differential equations, tostudy thetransmission dynamics of Lassa fever in Nigeria:

$$
\begin{aligned}
\frac{\mathrm{d} S_{h}}{\mathrm{~d} t} & =\pi_{h}+\xi_{h} R_{h}-\lambda_{h} S_{h}-\mu_{h} S_{h} \\
\frac{\mathrm{d} E_{h}}{\mathrm{~d} t} & =\lambda_{h} S_{h}-\left(\sigma_{h}+\mu_{h}\right) E_{h} \\
\frac{\mathrm{d} I_{h}}{\mathrm{~d} t} & =\sigma_{h} E_{h}-\left(\tau_{h}+\mu_{h}+\delta_{h}\right) I_{h} \\
\frac{\mathrm{d} R_{h}}{\mathrm{~d} t} & =\tau_{h} I_{h}-\left(\mu_{h}+\xi_{h}\right) R_{h} \\
\frac{\mathrm{d} S_{r}}{\mathrm{~d} t} & =\pi_{r}-\lambda_{r} S_{r}-\mu_{r} S_{r} \\
\frac{\mathrm{d} I_{r}}{\mathrm{~d} t} & =\lambda_{r} S_{r}-\mu_{r} I_{r}
\end{aligned}
$$

The model variables and parameters are presented in Table 1 and the flow diagram is depicted in Fig. 2.

\section{Basic properties of the model}

The basic properties of the Lassa fever model presented will be examined in this section. Since the mathematical model presented in the system of equations (1) describes the rate of change of different compartments of human and rodent populations, it will be epidemiologically meaningful if all its state variables are non-negative for all time $t$. In other words, the solutions of the model (1) with positive initial data will remain positive for all time $t>0$. It must be noted that, since the model presented describes the interaction between human and rodent populations, all the parameters of the model are assumed non-negative. Hence, we establish the following result.

\section{Positivity and boundedness of solutions}

Lemma 1 Let the initial data for the Lassa fever model (1) be $S_{h}(0)>0, E_{h}(0) \geq 0, I_{h}(0) \geq 0, R_{h} \geq 0, S_{r}(0)>0, I_{r}(0) \geq 0$. Then the solutions 


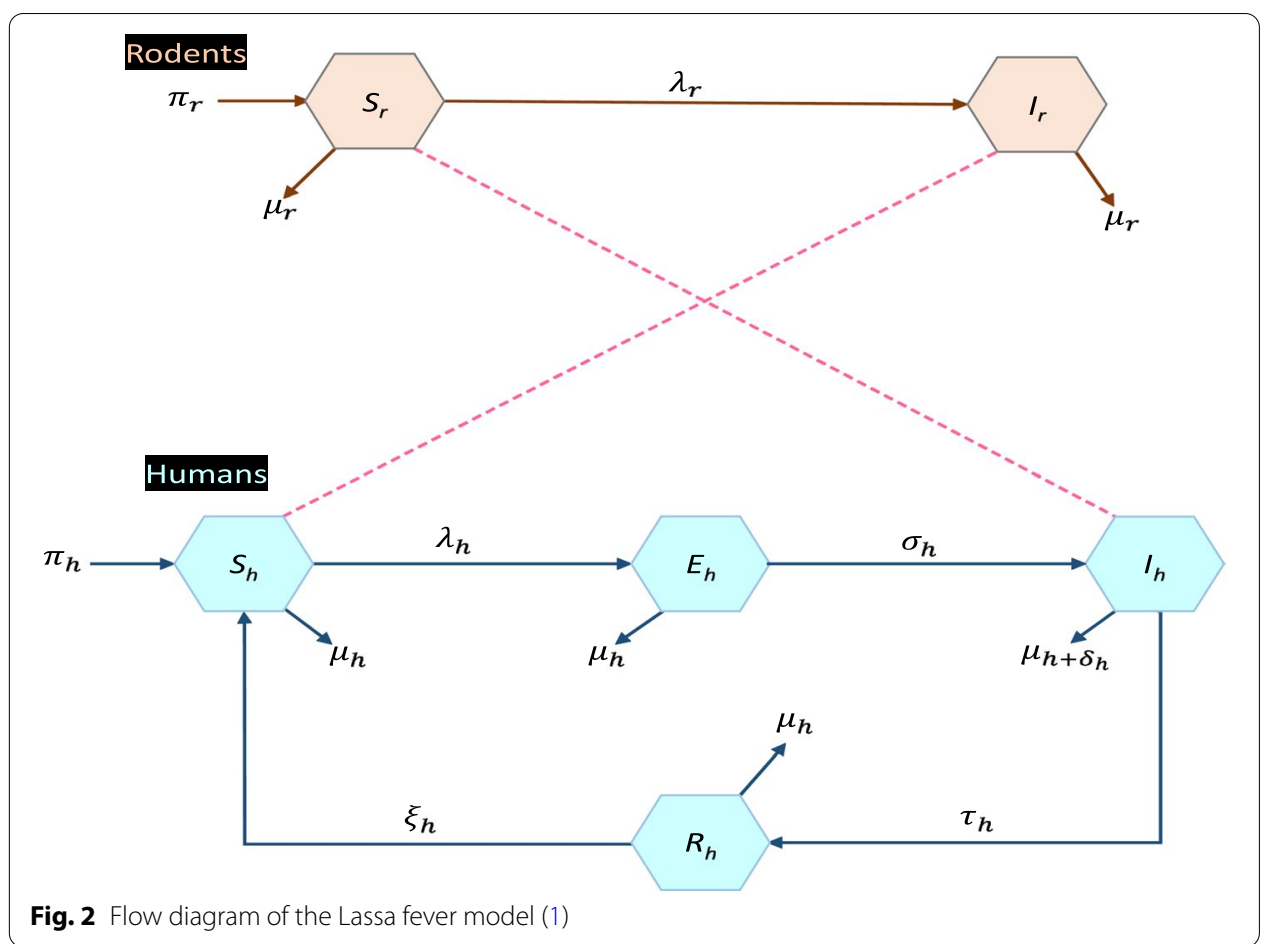

Table 1 Description of the variables and parameters of the Lassa fever model (1)

\begin{tabular}{ll}
\hline Variable & Description \\
\hline$S_{h}$ & Population of susceptible humans \\
$E_{h}$ & Population of exposed humans \\
$I_{h}$ & Population of infectious humans \\
$R_{h}$ & Population of recovered humans \\
$S_{r}$ & Population of susceptible rodents \\
$I_{r}$ & Population of infectious rodents \\
\hline Parameter & Description \\
\hline$\pi_{h}$ & Recruitment rate of humans through birth or immigration \\
$\xi_{h}$ & Immunity waning rate of humans \\
$\sigma_{h}$ & Disease progression rate from exposed to infectious human \\
$\tau_{h}$ & Recovery rate of infectious humans \\
$\mu_{h}$ & Natural death rate of humans \\
$\delta_{h}$ & Disease induced death rate for humans \\
$\beta_{h}$ & Transmission probability from human-to-human \\
$\beta_{r h}$ & Transmission probability from rodent-to-human \\
$\beta_{h r}$ & Transmission probability from human-to-rodent \\
$\beta_{r}$ & Transmission probability from rodent-to-rodent \\
$\pi_{r}$ & Recruitment rate of rodents through birth \\
$\mu_{r}$ & Natural death rate of rodents \\
\hline
\end{tabular}


$\left(S_{h}(t), E_{h}(t), I_{h}(t), R_{h}(t), S_{r}(t), I_{r}(t)\right)$ of the model with positive initial data, will remain positive for all time $t>0$.

Proof Let $t_{f}=\sup \left\{t>0: S_{h}(t)>0, E_{h}(t)>0, I_{h}(t)>0, R_{h}(t)>0, S_{r}(t)>0, I_{r}(t)>0 \in[0, t]\right\}$. Hence, $t_{f}>0$. It follows from the first equation of the model system (1), that

$$
\frac{\mathrm{d} S_{h}}{\mathrm{~d} t}=\pi_{h}+\xi_{h} R_{h}-\lambda_{h} S_{h}-\mu_{h} S_{h} \geq \pi_{h}-\lambda_{h} S_{h}-\mu_{h} S_{h}
$$

By employing the integrating factor method, equation (2) can be expressed as:

$$
\frac{\mathrm{d}}{\mathrm{d} t}\left(S_{h}(t) \exp \left[\mu_{h} t+\int_{0}^{t} \lambda_{h}(\omega) \mathrm{d} \omega\right]\right) \geq \pi_{h} \exp \left[\mu_{h} t+\int_{0}^{t} \lambda_{h}(\omega) \mathrm{d} \omega\right]
$$

Hence,

$$
S_{h}\left(t_{f}\right) \exp \left[\mu_{h} t_{f}+\int_{0}^{t_{f}} \lambda_{h}(\omega) \mathrm{d} \omega\right]-S_{h}(0) \geq \int_{0}^{t_{f}} \pi_{h}\left(\exp \left[\mu_{h} \theta+\int_{0}^{\theta} \lambda_{h}(\omega) \mathrm{d} \omega\right]\right) \mathrm{d} \theta
$$

so that,

$$
\begin{aligned}
S_{h}\left(t_{f}\right) & \geq S_{h}(0) \exp \left[-\mu_{h} t_{f}-\int_{0}^{t_{f}} \lambda_{h}(\omega) \mathrm{d} \omega\right] \\
& +\exp \left[-\mu_{h} t_{f}-\int_{0}^{t_{f}} \lambda_{h}(\omega) \mathrm{d} \omega\right] \times \int_{0}^{t_{f}} \pi_{h}\left(\exp \left[\mu_{h} \theta+\int_{0}^{\theta} \lambda_{h}(\omega) \mathrm{d} \omega\right]\right) \mathrm{d} \theta>0 .
\end{aligned}
$$

In the same way, the remaining state variables $E_{h}(t) \geq 0, I_{h}(t) \geq 0, R_{h}(t) \geq 0, S_{r}(t)>0$, and $I_{r}(t) \geq 0$ for all time $t>0$. Hence, all the solutions of model (1) remain positive for all non-negative initial conditions.

\section{Invariant region}

Here, we show the invariant regions for the given Lassa fever model (1). Consider the biologically feasible region consisting of $\mathcal{D}=\mathcal{D}_{h} \times \mathcal{D}_{r} \in \mathcal{R}_{+}^{4} \times \mathcal{R}_{+}^{2}$ with

$$
\mathcal{D}_{h}=\left\{S_{h}, E_{h}, I_{h}, R_{h} \in \mathcal{R}_{+}^{4}: N_{h} \leq \frac{\pi_{h}}{\mu_{h}}\right\}
$$

and

$$
\mathcal{D}_{r}=\left\{S_{r}, I_{r} \in \mathcal{R}_{+}^{2}: N_{r} \leq \frac{\pi_{r}}{\mu_{r}}\right\}
$$

It can be shown that the set $\mathcal{D}$ is a positively invariant set of the model system (1). This implies that all the solution trajectories initiated at any point of the non-negative region $\mathcal{R}_{+}^{6}$ will enter the feasible region $\mathcal{D}$ and remain there for all time $t$. The result is summarized in the following Lemma. 
Lemma 2 The biological feasible region $\mathcal{D}=\mathcal{D}_{h} \cup \mathcal{D}_{r} \subset \mathcal{R}_{+}^{4} \times \mathcal{R}_{+}^{2}$ of the Lassa fever model (1) is positively invariant with non-negative initial conditions in $\mathcal{R}_{+}^{6}$.

Proof The summation of the human and rodent populations $N_{h}$ and $N_{r}$ of the Lassa fever model (1) result to

$$
\begin{aligned}
& \frac{\mathrm{d} N_{h}(t)}{\mathrm{d} t}=\pi_{h}-\mu_{h} N_{h}(t)-\delta_{h} I_{h}(t) \\
& \frac{\mathrm{d} N_{r}(t)}{\mathrm{d} t}=\pi_{r}-\mu_{r} N_{r}(t)
\end{aligned}
$$

Thus,

$$
\frac{\mathrm{d} N_{h}(t)}{\mathrm{d} t} \leq \pi_{h}-\mu_{h} N_{h}(t), \quad \text { and } \quad \frac{\mathrm{d} N_{r}(t)}{\mathrm{d} t}=\pi_{r}-\mu_{r} N_{r}(t)
$$

Solving the above yields $\quad N_{h}(t) \leq N_{h}(0) e^{-\mu_{h} t}+\frac{\pi_{h}}{\mu_{h}}\left(1-e^{-\mu_{h} t}\right) \quad$ and $N_{r}(t)=N_{r}(0) e^{-\mu_{r} t}+\frac{\pi_{r}}{\mu_{r}}\left(1-e^{-\mu_{r} t}\right)$. It follows that $N_{h}(t) \rightarrow \frac{\pi_{h}}{\mu_{h}}$ and $N_{r}(t) \rightarrow \frac{\pi_{r}}{\mu_{r}}$ as $t \rightarrow \infty$. In particular, $N_{h}(t) \leq \frac{\pi_{h}}{\mu_{h}}$ if the total human population at the initial time $N_{h}(0) \leq \frac{\pi_{h}}{\mu_{h}}$. Similarly, $N_{r}(t) \leq \frac{\pi_{r}}{\mu_{r}}$ if the total rodent population at the initial time $N_{r}(0) \leq \frac{\pi_{r}}{\mu_{r}}$. Thus, the region $\mathcal{D}$ is positively invariant.

Hence, it is suitable to study the transmission dynamics of Lassa fever using model (1) in the biological feasible region $\mathcal{D}$, for which the model is said to be epidemiologically and mathematically well-posed [38, 39].

\section{Mathematical analysis of the model}

In this section, we critically analyze model (1) by determining the existence of the steadystate solutions. This includes the existence of the disease-free equilibrium (henceforth called Lassa fever-free equilibrium) and the endemic equilibrium. We further investigate the local and global stability of the equilibria. Furthermore, we investigate the nature of bifurcation the model exhibit.

\section{Existence and stability of Lassa fever-free equilibrium}

Lassa fever-free equilibrium points are the steady-state solution in the absence of Lassa fever infection. Thus, the Lassa fever-free equilibrium point for model (1) implies that $E_{h}=I_{h}=I_{r}=0$. Hence, by solving the systems of equations simultaneously (1), the Lassa fever-free equilibrium denoted by $\mathcal{E}_{0}$, is obtained as

$$
\mathcal{E}_{0}=\left(S_{h}^{*}, E_{h}^{*}, I_{h}^{*}, R_{h}^{*}, S_{r}^{*}, I_{r}^{*}\right)=\left(\frac{\pi_{h}}{\mu_{h}}, 0,0,0, \frac{\pi_{r}}{\mu_{r}}, 0\right)
$$

To investigate the local stability of the Lassa fever-free equilibrium, we compute the basic reproduction number $\mathcal{R}_{0}$ by using the next generation operator method on the model system (1). Following the approach in [22, 40], the Jacobian matrices $F$ and $V$, for the new infection terms and the remaining transfer terms are given by 


$$
F=\left(\begin{array}{ccc}
0 & \beta_{h} & \beta_{r h} \\
0 & 0 & 0 \\
0 & \frac{\beta_{h S_{r}^{*}}^{*}}{S_{h}^{*}} & \beta_{r}
\end{array}\right) \quad \text { and } \quad V=\left(\begin{array}{ccc}
k_{1} & 0 & 0 \\
-\sigma_{h} & k_{2} & 0 \\
0 & 0 & \mu_{r}
\end{array}\right)
$$

where $k_{1}=\sigma_{h}+\mu_{h}$, and $k_{2}=\tau_{h}+\mu_{h}+\delta_{h}$. The next generation matrix (NGM) with large domain $K_{L}$ is given below as

$$
K_{L}=F V^{-1}=\left(\begin{array}{ccc}
\frac{\beta_{h} \sigma_{h}}{k_{1} k_{2}} & \frac{\beta_{h}}{k_{2}} & \frac{\beta_{r h}}{\mu_{r}} \\
0 & 0 & 0 \\
\frac{\beta_{h r} r_{r}^{*} \sigma_{h}}{S_{h}^{*} k_{1} k_{2}} & \frac{\beta_{h r} S_{r}^{*}}{S_{h}^{*} k_{2}} & \frac{\beta_{r}}{\mu_{r}}
\end{array}\right)
$$

It can be seen from the model that, among the three infected states, there are only two that are states-at-infection. This can also be seen by looking at matrix $F$ and observing that the entire second row contains zeros. Hence, the NGM $K$ for the small domain is therefore two-dimensional. Thus, using the approach of [41] with an auxiliary matrix $E$, the NGM $K$ is obtained as

$$
K=E^{T} K_{L} E=E^{T} F V^{-1} E=\left(\begin{array}{cc}
\frac{\beta_{h} \sigma_{h}}{k_{1} k_{2}} & \frac{\beta_{r h}}{\mu_{r}} \\
\frac{\beta_{h h} S_{r}^{*} \sigma_{h}}{S_{h}^{*} k_{1} k_{2}} & \frac{\beta_{r}}{\mu_{r}}
\end{array}\right)=\left(\begin{array}{cc}
\mathcal{R}_{h} & \mathcal{R}_{r h} \\
\mathcal{R}_{h r} & \mathcal{R}_{r}
\end{array}\right)
$$

Thus, the characteristic polynomial of the matrix $K$ is obtained as

$$
\lambda^{2}-\left(\mathcal{R}_{h}+\mathcal{R}_{r}\right) \lambda+\left(\mathcal{R}_{h} \mathcal{R}_{r}-\mathcal{R}_{h r} \mathcal{R}_{r h}\right)=0
$$

where

$$
\mathcal{R}_{h}=\frac{\beta_{h} \sigma_{h}}{k_{1} k_{2}}, \quad \mathcal{R}_{r}=\frac{\beta_{r}}{\mu_{r}}, \quad \mathcal{R}_{h r}=\frac{\beta_{h r} S_{r}^{*} \sigma_{h}}{S_{h}^{*} k_{1} k_{2}}, \quad \mathcal{R}_{r h}=\frac{\beta_{r h}}{\mu_{r}} .
$$

It follows that the basic reproduction number for the model (1), which is the spectral radius of $K$ given by $\mathcal{R}_{0}=\rho(K)$, is obtained as

$$
\mathcal{R}_{0}=\frac{1}{2}\left\{\left(\mathcal{R}_{h}+\mathcal{R}_{r}\right)+\sqrt{\left(\mathcal{R}_{h}+\mathcal{R}_{r}\right)^{2}-4\left(\mathcal{R}_{h} \mathcal{R}_{r}-\mathcal{R}_{h r} \mathcal{R}_{r h}\right)}\right\}
$$

Further simplification of (8) result to

$$
\mathcal{R}_{0}=\frac{1}{2}\left\{\left(\mathcal{R}_{h}+\mathcal{R}_{r}\right)+\sqrt{\left(\mathcal{R}_{h}-\mathcal{R}_{r}\right)^{2}+4 \mathcal{R}_{\Delta}^{2}}\right\}
$$

where $\mathcal{R}_{h}, \mathcal{R}_{r}$, and $\mathcal{R}_{\Delta}=\sqrt{\mathcal{R}_{h r} \mathcal{R}_{r h}}$ are the reproduction numbers for human-tohuman, rodent-to-rodent transmission and vectorial transmission respectively.

The basic reproduction number is a threshold quantity that measures the spread potential of disease in a given population. Epidemiologically, it measures the average 
number of secondary infections a single infected individual can generate in a population that is completely susceptible. In other words, the threshold quantity $\mathcal{R}_{0}$ given in (9) measures the average number of Lassa fever infections that a Lassa fever infected individual can generate in an entirely susceptible population. It is imperative to mention that, the reproduction number for the model (1) is a composition of the reproduction number of human-to-human transmission $\mathcal{R}_{h}$, rodent-to-rodent transmission $\mathcal{R}_{r}$, and vectorial transmission $\mathcal{R}_{h r}, \mathcal{R}_{r h}$ because the model includes the biological possibilities of infection transfer between the two interacting host. Hence, epidemiologically, $\mathcal{R}_{h}$ measure the average number of secondary infections a single infectious human can produce during an infectious period. Similarly, $\mathcal{R}_{r}$ measure the average number of secondary infections a single infectious rodent can generate during an infectious period. Since $\beta_{h r}$, and $\beta_{r h}$ are the transmission probability from human-to-rodent, and rodent-to-human respectively, then $\mathcal{R}_{h r}$ measure the average number of secondary infection of rodents a single infectious human can generate over its infectious period, while $\mathcal{R}_{r h}$ measure the average number of secondary infection of humans a single infectious rodent can generate during the infection period. In general, an increase in any of the reproduction number can upsurge the risk of Lassa fever occurrence in the human population, since the growth of any of the infectious host (either humans or rodents) can increase the spread of infection in the human populace if adequate and effective control mechanism is not utilized by the population. Next, we shall investigate the stability of the Lassa fever-free equilibrium $\mathcal{E}_{0}$.

\section{Local stability of Lassa fever-free equilibrium}

We analyze the local stability of Lassa fever-free equilibrium of the model system (1) by using the basic reproduction number $\mathcal{R}_{0}$ in the following theorem as described in [38]. The proof is provided in "Appendix Proof of Theorem 1".

Theorem 1 The Lassa fever-free equilibrium $\mathcal{E}_{0}$, of the model (1) is locally asymptotically stable in the biological feasible region $\mathcal{D}$ if $\mathcal{R}_{0}<1$ and unstable if $\mathcal{R}_{0}>1$.

\section{Global stability of Lassa fever-free equilibrium}

Here, we further investigate the global stability of the Lassa fever-free equilibrium $\mathcal{E}_{0}$ of the model system (1), by using the technique implemented in [42]. Firstly, we re-write the Lassa fever model (1) in the form

$$
\begin{aligned}
& \frac{\mathrm{d} X}{\mathrm{~d} t}=F(X, Z) \\
& \frac{\mathrm{d} Z}{\mathrm{~d} t}=G(X, Z), \quad G(X, 0)=0
\end{aligned}
$$

where $X=\left(S_{h}, R_{h}, S_{r}\right)$ is the uninfected population, and $Z=\left(E_{h}, I_{h}, I_{r}\right)$ is the infected population with the component of $(X, Z) \in \mathcal{R}^{3}$. The Lassa fever-free equilibrium is obtained as 


$$
\mathcal{E}_{0}^{*}=\left(X^{*}, 0\right)=\left(\frac{\pi_{h}}{\mu_{h}}, 0, \frac{\pi_{r}}{\mu_{r}}\right)
$$

For the point $\mathcal{E}_{0}^{*}=\left(X^{*}, 0\right)$ to be globally asymptotically stable, the following conditions must be satisfied

(C1) : For $\frac{\mathrm{d} X}{\mathrm{~d} t}=F(X, 0), X^{*}$ is globally asymptotically stable (GAS),

$(C 2): G(X, Z)=Q Z-\hat{G}(X, Z)$ with $\hat{G}(X, Z) \geq 0$ for $(X, Z) \in \mathcal{D}$

where $Q=B_{Z} G\left(X^{*}, 0\right)$ is an M-matrix (the off-diagonal elements of $B$ are non-negative) and $\mathcal{D}$ is the feasible region where the model makes biological sense. If the model system (1) satisfies the conditions given above, then the following result holds. The proof is provided in "Appendix Proof of Theorem 2".

Theorem 2 The fixed point $\mathcal{E}_{0}^{*}=\left(X^{*}, 0\right)$ is globally asymptotically stable $(G A S)$ equilibrium of model system (1), if $\mathcal{R}_{0}<1$ (locally asymptotically stable) and the conditions (C1) and (C2) are satisfied.

The above result infers that, regardless of the initial sizes of the sub-populations of the system, Lassa fever eradication is possible whenever the reproduction number is less than unity. We illustrate this theorem numerically in Fig. 10.

\section{Existence and stability of endemic equilibria}

Here, we investigate the existence and stability of the endemic equilibrium for the model (1). Lassa fever endemic equilibrium points are the steady-state solution where there is presence of Lassa fever infection in the population. We let $\mathcal{E}_{1}=\left(S_{h}^{* *}, E_{h}^{* *}, I_{h}^{* *}, R_{h}^{* *}, S_{r}^{* *}, I_{r}^{* *}\right)$ represents the Lassa fever-present equilibrium. Setting the right-hand sides of the systems of equations in (1) to zero and solving simultaneously in terms of the associated form of infection yields

$$
\begin{aligned}
S_{h}^{* *} & =\frac{\pi_{h} k_{1} k_{2} k_{3}}{k_{1} k_{2} k_{3} \lambda_{h}^{* *}+k_{1} k_{2} k_{3} \mu_{h}-\lambda_{h}^{* *} \sigma_{h} \tau_{h} \xi_{h}} \\
E_{h}^{* *} & =\frac{\lambda_{h}^{* *} \pi_{h} k_{2} k_{3}}{k_{1} k_{2} k_{3} \lambda_{h}^{* *}+k_{1} k_{2} k_{3} \mu_{h}-\lambda_{h}^{* *} \sigma_{h} \tau_{h} \xi_{h}} \\
I_{h}^{* *} & =\frac{\lambda_{h}^{* *} \pi_{h} \sigma_{h} k_{3}}{k_{1} k_{2} k_{3} \lambda_{h}^{* *}+k_{1} k_{2} k_{3} \mu_{h}-\lambda_{h}^{* *} \sigma_{h} \tau_{h} \xi_{h}} \\
R_{h}^{* *} & =\frac{\lambda_{h}^{* *} \pi_{h} \sigma_{h} \tau_{h}}{k_{1} k_{2} k_{3} \lambda_{h}^{* *}+k_{1} k_{2} k_{3} \mu_{h}-\lambda_{h}^{* *} \sigma_{h} \tau_{h} \xi_{h}} \\
S_{r}^{* *} & =\frac{\pi_{r}}{\lambda_{r}^{* *}+\mu_{r}}, \quad I_{r}^{* *}=\frac{\lambda_{r}^{* *} \pi_{r}}{\mu_{r}\left(\lambda_{r}^{* *}+\mu_{r}\right)}
\end{aligned}
$$

where the force of infection are given as 


$$
\lambda_{h}^{* *}=\frac{\beta_{r h} I_{r}^{* *}}{N_{h}^{* *}}+\frac{\beta_{h} I_{h}^{* *}}{N_{h}^{* *}}, \quad \text { and } \quad \lambda_{r}^{* *}=\frac{\beta_{h r} I_{h}^{* *}}{N_{h}^{* *}}+\frac{\beta_{r} I_{r}^{* *}}{N_{r}^{* *}}
$$

Substituting the expression (12) into the force of infection (13) at steady state yields the following polynomial

$$
\lambda_{h}^{* *}\left\{a_{1}\left(\lambda_{h}^{* *}\right)^{4}+a_{2}\left(\lambda_{h}^{* *}\right)^{3}+a_{3}\left(\lambda_{h}^{* *}\right)^{2}+a_{4} \lambda_{h}^{* *}-a_{5}\right\}=0
$$

The coefficients $a_{i}$, for $i=1 \ldots, 5$ of the polynomial are given in "Appendix Coefficients of polynomial (14)". Clearly, $\lambda_{h}^{* *}=0$ is a solution. The coefficient $a_{1}$ is positive while the sign of $a_{5}$ depends on the values of respective reproduction number, such that if $\left\{\mathcal{R}_{h}, \mathcal{R}_{r}, \mathcal{R}_{h r}, \mathcal{R}_{h r} \in \mathcal{R}_{0}>1\right\}$, then $a_{5}>0$ such that there is at least one sign change in the sequence of coefficients $a_{1}, \ldots a_{5}$. Thus, by Descartes rule of signs, there exists at least one positive real root for (14) aside from the root $\lambda_{h}^{* *}=0$, whenever $\mathcal{R}_{0}>1$. Therefore, the following result is established.

Theorem 3 The model system (1) has at least one endemic equilibrium whenever $\mathcal{R}_{0}>1$.

\section{Bifurcation analysis}

Following Theorem 1, it is imperative to re-state that, whenever the reproduction number of the model (1) is greater than unity $\mathcal{R}_{0}>1$, the asymptotic local stability of the Lassa fever-free equilibrium will undergo a trade-off with the asymptomatic local stability of the endemic equilibrium. Hence, in this section, we will investigate the criteria for the trade-off between the asymptomatic local stability of the Lassa fever-free equilibrium and asymptomatic local stability of the endemic equilibrium, as the threshold quantity crosses unity. In other words, we will show the conditions under which model (1) undergo supercritical or subcritical (forward or backward) bifurcation. By employing the Center Manifold Theory of bifurcation analysis described in [27], we write the Lassa fever model (1) in the vector form

$$
\frac{\mathrm{d} X}{\mathrm{~d} t}=F(X)
$$

where $X=\left(x_{1}, x_{2}, x_{3}, x_{4}, x_{5}, x_{6}\right)^{T}$ and $F=\left(f_{1}, f_{2}, f_{3}, f_{4}, f_{5}, f_{6}\right)^{T}$. We further modify the variables be setting

$$
S_{h}=x_{1}, \quad E_{h}=x_{2}, \quad I_{h}=x_{3}, \quad R_{h}=x_{4}, \quad S_{r}=x_{5}, \quad I_{r}=x_{6}
$$

such that the total human and rodent populations are respectively given as

$$
N_{h}=x_{1}+x_{2}+x_{3}+x_{4}, \quad \text { and } \quad N_{r}=x_{5}+x_{6}
$$

Hence, following the above transformation, the transformed model (1) is given as 


$$
\begin{aligned}
& \frac{\mathrm{d} x_{1}}{\mathrm{~d} t}=f_{1}=\pi_{h}+\xi_{h} x_{4}-\lambda_{h} x_{1}-\mu_{h} x_{1} \\
& \frac{\mathrm{d} x_{2}}{\mathrm{~d} t}=f_{2}=\lambda_{h} x_{1}-\left(\sigma_{h}+\mu_{h}\right) x_{2} \\
& \frac{\mathrm{d} x_{3}}{\mathrm{~d} t}=f_{3}=\sigma_{h} x_{2}-\left(\tau_{h}+\mu_{h}+\delta_{h}\right) x_{3} \\
& \frac{\mathrm{d} x_{4}}{\mathrm{~d} t}=f_{4}=\tau_{h} x_{3}-\left(\mu_{h}+\xi_{h}\right) x_{4} \\
& \frac{\mathrm{d} x_{5}}{\mathrm{~d} t}=f_{5}=\pi_{r}-\lambda_{r} x_{5}-\mu_{r} x_{5} \\
& \frac{\mathrm{d} x_{6}}{\mathrm{~d} t}=f_{6}=\lambda_{r} x_{5}-\mu_{r} x_{6}
\end{aligned}
$$

with the associated force of infection given as

$$
\lambda_{h}=\frac{\beta_{r h} x_{6}+\beta_{h} x_{3}}{x_{1}+x_{2}+x_{3}+x_{4}}, \quad \lambda_{r}=\frac{\beta_{h r} x_{3}}{x_{1}+x_{2}+x_{3}+x_{4}}+\frac{\beta_{r} x_{6}}{x_{5}+x_{6}}
$$

Suppose that $\beta_{r h}^{*}$ is chosen as the bifurcation parameter, solving (8) at $\mathcal{R}_{0}=1$, the parameter $\beta_{r h}=\beta_{r h}^{*}$ is obtained as

$$
\beta_{r h}:=\beta_{r h}^{*}=\frac{\pi_{h} \mu_{r}\left\{\mu_{r} k_{1} k_{2}-\left(\beta_{h} \sigma_{h} \mu_{r}+\beta_{r} \beta_{h} \sigma_{h}+\beta_{r} k_{1} k_{2}\right)\right\}}{\beta_{h r} \pi_{r} \sigma_{h} \mu_{h}}
$$

The Jacobian of system (16), evaluated at Lassa fever-free $\left(\mathcal{E}_{0}^{\Delta}=x_{1}^{*}, 0,0,0, x_{5}^{*}, 0\right)$ with $\beta_{r h}=\beta_{r h}^{*}$ denoted by $\mathcal{J}\left(\mathcal{E}_{0}^{\triangle}, \beta_{r h}^{*}\right)$ is given by

$$
\mathcal{J}\left(\mathcal{E}_{0}^{\Delta}, \beta_{r h}^{*}\right)=\left(\begin{array}{cccccc}
-\mu_{h} & 0 & -\beta_{h} & \xi_{h} & 0 & -\beta_{r h}^{*} \\
0 & -k_{1} & \beta_{h} & 0 & 0 & \beta_{r h}^{*} \\
0 & \sigma_{h} & -k_{2} & 0 & 0 & 0 \\
0 & 0 & \tau_{h} & -k_{3} & 0 & 0 \\
0 & 0 & -\frac{x_{5}^{*} \beta_{h r}}{x_{1}^{*}} & 0 & -\mu_{r} & -\beta_{r} \\
0 & 0 & \frac{x_{5}^{*} \beta_{h r}}{x_{1}^{*}} & 0 & 0 & -\mu_{r}+\beta_{r}
\end{array}\right)
$$

The Jacobian matrix (18) has a right eigenvector (associated with the zero eigenvalues) given by $\mathbf{w}=\left(w_{1}, w_{2}, w_{3}, w_{4}, w_{5}, w_{6}\right)^{T}$, where

$$
\begin{aligned}
& w_{1}=\left(\frac{x_{1}^{*} \mu_{r}\left(1-\mathcal{R}_{r}\right)\left(\tau_{h} \xi_{h}-\beta_{h} k_{3}\right)-x_{5}^{*} \beta_{h r}}{x_{1}^{*} \mu_{r} \mu_{h} k_{3}\left(1-\mathcal{R}_{r}\right)}\right) w_{3} ; \quad w_{2}=\frac{w_{3} k_{2}}{\sigma_{h}} ; \quad w_{3}=w_{3}>0 ; \\
& w_{4}=\frac{w_{3} \tau_{h}}{k_{3}} ; \quad w_{5}=-\frac{w_{3} x_{5}^{*}}{x_{1}^{*} \mu_{r}\left(1-\mathcal{R}_{r}\right)} ; \quad w_{6}=\frac{w_{3} x_{5}^{*} \beta_{h r}}{x_{1}^{*} \mu_{r}\left(1-\mathcal{R}_{r}\right)}
\end{aligned}
$$

Similarly, the Jacobian matrix (18) has a left eigenvector (associated with the zero eigenvalues) given by $\mathbf{v}=\left(v_{1}, v_{2}, v_{3}, v_{4}, v_{5}, v_{6}\right)^{T}$, where 


$$
v_{1}=0 ; \quad v_{2}=\frac{v_{3} \sigma_{h}}{k_{1}} ; \quad v_{3}=v_{3}>0 ; \quad v_{4}=0 ; \quad v_{5}=0 ; \quad v_{6}=\frac{v_{3} \beta_{r h}^{*} \sigma_{h}}{k_{1} \mu_{r}\left(1-\mathcal{R}_{r}\right)}
$$

\section{Computation of bifurcation coefficient $a$ and $b$}

The direction of the bifurcation at $\mathcal{R}_{0}=1$ is determined by the signs of bifurcation coefficients $a$ and $b$, obtained by computing the associated non-zero partial derivative of $F(X)$ (evaluated at the disease free equilibrium $\mathcal{E}_{0}^{\Delta}$ ). Thus, the coefficient of $a$ is given as

$$
\begin{aligned}
a & =\sum_{k, i, j=1}^{6} v_{k} w_{i} w_{j} \frac{\partial^{2} f_{k}}{\partial x_{i} \partial x_{j}}(0,0) \\
& =\frac{2\left(m_{1}-m_{2}\right)}{x_{1}^{* 2} x_{5}^{*}}
\end{aligned}
$$

where

$$
\begin{aligned}
& m_{1}=x_{1}^{*} x_{5}^{*}\left\{m_{3}\left(\beta_{h} w_{3} v_{1}+\beta_{r h}^{*} v_{1} w_{6}\right)+\beta_{h r} w_{3} w_{5} v_{6}\right\}+\beta_{h r} v_{5} w_{3} x_{5}^{* 2}\left(w_{1}+m_{3}\right)+\beta_{r} x_{1}^{* 2} v_{5} w_{6}^{2} \\
& m_{2}=x_{1}^{*} x_{5}^{*}\left\{m_{3}\left(\beta_{h} w_{3} v_{2}+\beta_{r h}^{*} v_{2} w_{6}\right)+\beta_{h r} w_{3} w_{5} v_{5}\right\}+\beta_{h r} v_{6} w_{3} x_{5}^{* 2}\left(w_{1}+m_{3}\right)+\beta_{r} x_{1}^{* 2} v_{6} w_{6}^{2} \\
& m_{3}=w_{2}+w_{3}+w_{4}
\end{aligned}
$$

Similarly, the bifurcation coefficient $b$ is obtained as follows

$$
\begin{aligned}
b & =\sum_{k, i=1}^{6} v_{k} w_{i} \frac{\partial^{2} f_{k}}{\partial x_{i} \partial \beta_{r h}^{*}}(0,0) \\
& =w_{6}\left(v_{2}-v_{1}\right)>0
\end{aligned}
$$

Since all the parameters of model (1) are non-negative and $v_{1}=0$, it can be shown that the inequality (20) holds if $\mathcal{R}_{r}<1$. It follows from Theorem 4.1 in [27] that the Lassa fever model (1) will exhibit a subcritical (backward) bifurcation if the coefficient $a$ given by (19) is positive. This implies that $m_{1}>m_{2}$ must be satisfied. Hence, the following result will be established.

Theorem 4 The Lassa fever model (1) undergoes a subcritical (backward) bifurcation as $\mathcal{R}_{0}$ crosses unity, whenever the coefficient $a>0$ and $b>0$.

Backward bifurcation (BB) occurs when a small positive unstable equilibrium appears while the disease-free equilibrium (DFE) and a larger positive equilibrium are locally asymptotically stable when the threshold quantity $\mathcal{R}_{0}$ is less than unity. In other words, BB occurs when a stable DFE and a stable endemic equilibrium coexist under some given values for which $\mathcal{R}_{0}$ is less than unity. The backward bifurcation phenomenon suggests that the epidemiological condition of having the reproduction number less than unity to eliminate a disease although necessary is no longer enough for the effective control of the disease in the population. Hence, the effective control of Lassa fever in the population is difficult, since disease control when $\mathcal{R}_{0}<1$ is dependent on the initial sizes of the sub-populations. We further explore the condition for which system (1) undergo supercritical bifurcation. It must be noted that the Lassa fever model (1) will exhibit a forward bifurcation if the coefficient $a$ given by (19) is negative. This implies that $m_{1}<m_{2}$ must be satisfied. Thus, the following result will be established. 


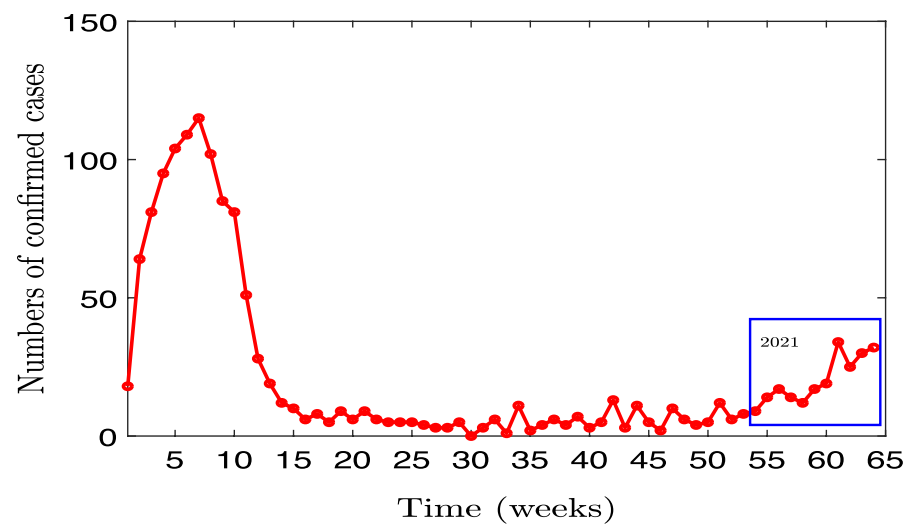

Fig. 3 Weekly reported number of confirmed Lassa fever cases in Nigeria from first week January 2020 to eleventh week in 2021, obtained from NCDC database [13]

Theorem 5 The Lassa fever model (1) undergoes a supercritical (forward) bifurcation as $\mathcal{R}_{0}$ crosses unity, whenever the coefficient $a<0$ and $b>0$.

A system exhibits a forward bifurcation when the disease-free equilibrium losses its stability due to an introduction of a small positive asymptomatically stable equilibrium. Epidemiologically, the result above implies that a small inflow of individuals with Lassa fever infection into an entirely susceptible population will lead to a continuance of Lassa fever in the populace, whenever the reproduction number is less than unity. In other words, the exchange of the local asymptotic stability of the equilibria depends on the initial number of Lassa fever infectious individuals in the population. It must be noted that the transfer of the local asymptotic stability of the equilibria is independent of the initial sizes of the sub-populations. This can be proved by establishing the global asymptomatic stability of the disease-free equilibrium (see "section Global stability of Lassa fever-free equilibrium").

\section{Parameter estimation and data fitting}

Estimating parameter values is very vital for precise prediction in an epidemiological study. To make the prediction of model results meaningful, it is more valuable to validate the formulated model with real-life data. This can be achieved by fitting the proposed model with the real data, to inform the population of the degree of precision and validation of the model's ability on predicting a realistic outcome. In this section, we parameterized model (1) by using the Lassa fever reported cases from Nigeria. We used the data for a period from the first week in January 2020 through the eleventh week in 2021, obtained through the Nigeria Centre for Disease Control (NCDC) database [13]. The number of cumulative confirmed cases for this period is depicted in Fig. 3. The blue box captioned the trend of the cumulative confirmed cases from the beginning of the year 2021. It should be noted that the confirmed cases of Lassa fever are increasing as the week progresses. Thus, it is important to provide adequate control strategies to curtail the spread of Lassa fever in the population. We obtained our parameter values through two different strategies. The Lassa fever model presented 
in this work contains twelve parameters and six of the parameters are estimated as follows; the natural death rate of human $\mu_{h}$ is a demographic parameter estimated by

$$
\mu_{h}=\frac{1}{\mu_{0}}
$$

where $\mu_{0}$ is the average life expectancy of humans. The average life expectancy of humans in Nigeria as presented in [43] is 60.45 years. In addition, the total human population $\left(N_{h}\right)$ of Nigerians is recorded as 214, 028, 302 [43], hence since we assumed by the invariant region that $N_{h}=\frac{\pi_{h}}{\mu_{h}}$, we estimated the recruitment rate by $N_{h} \times \mu_{h}$. Similarly, the natural death of rodent $\mu_{r}$ is estimated by $\mu_{r}=\frac{1}{\mu_{0}}$, where $\mu_{0}=1$ year is the average life expectancy of natal multimammate rat $[2,10]$. Furthermore, we assume the total population of rodents to be $N_{r}=30,000$, so that the recruitment rate of rodents is obtained by $N_{r} \times \mu_{r}$. According to [44], the incubation period of Lassa fever ranges between $6-21$ days, thus we estimate the disease progression rate from exposed human to infectious human $\sigma_{h}$ as 0.5185 per week. Lastly, using the reported death cases due to Lassa fever and reported confirmed cases denoted as $(D, I)$ respectively, the Lassa feverinduced death rate $\delta_{h}$ is obtained by

$$
\delta_{h}=\frac{\sum_{t=1}^{n} D_{t}}{\sum_{t=1}^{n} I_{t}}
$$

where $t=1,2, \ldots, n$ is the time measured in weeks and $n=64$ is the total number of weeks reported in the used data. All parameter value units are provided in per-week. To obtain the remaining six parameter values, we fit the Lassa fever model (1) to the obtained cumulative number of cases reported in [13]. The model fitting was implemented by using the standard nonlinear least square method in MATLAB-R2017b. All the parameter values estimated and fitted are tabulated in Table 2, while Fig. 4 depicts the data fitting of the observed cumulative confirmed cases. Using the parameter values, the reproduction number given in (9) is estimated as $\mathcal{R}_{0}=1.32$. We further use the parameter values to perform the sensitivity analysis and to simulate the different

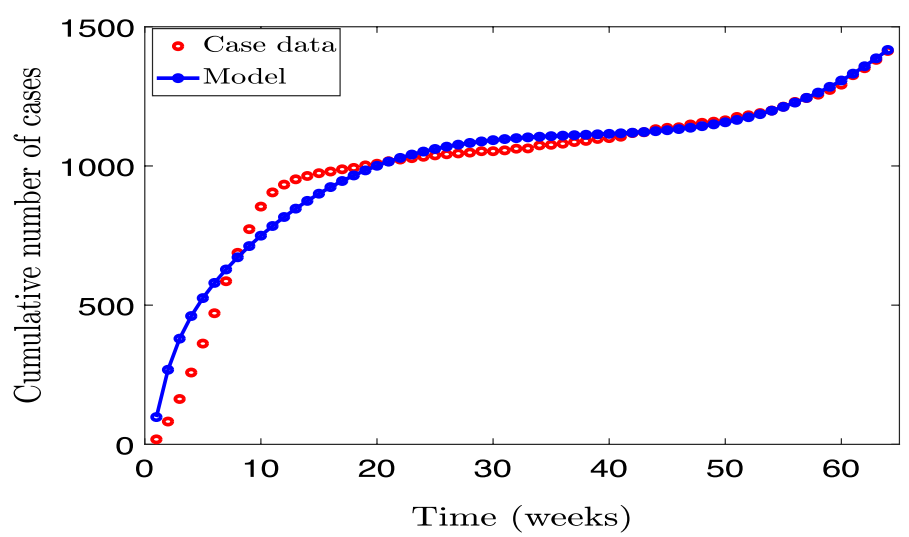

Fig. 4 Data fitting of the Lassa fever model (1) using cumulative confirmed cases data for Nigeria, from first week January 2020 to eleventh week in 2021. Reported cases are obtained from NCDC database [13] 
Table 2 Parameter values for the Lassa fever model (1)

\begin{tabular}{llll}
\hline Parameter & Description & Value & Source \\
\hline$\pi_{h}$ & Recruitment rate of humans through birth or immigration & 68,088 & Estimated \\
$\sigma_{h}$ & Disease progression rate from exposed to infectious human & 0.5185 & Estimated \\
$\mu_{h}$ & Natural death rate of humans & 0.0003 & Estimated \\
$\delta_{h}$ & Disease induced death rate for humans & 0.1323 & Estimated \\
$\xi_{h}$ & Immunity waning rate of humans & 0.3278 & Fitted \\
$\tau_{h}$ & Recovery rate of infectious humans & 0.0027 & Fitted \\
$\beta_{h}$ & Transmission probability from human-to-human & 0.1250 & Fitted \\
$\beta_{r h}$ & Transmission probability from rodent-to-human & 0.0509 & Fitted \\
$\beta_{h r}$ & Transmission probability from human-to-rodent & 0.0137 & Fitted \\
$\beta_{r}$ & Transmission probability from rodent-to-rodent & 0.0254 & Fitted \\
$\pi_{r}$ & Recruitment rate of rodents through birth & 577 & Estimated \\
$\mu_{r}$ & Natural death rate of rodents & 0.0192 & Estimated \\
\hline
\end{tabular}

scenarios of Lassa fever dynamics in the population, to provide precise predictions or recommendations for health care practitioners.

\section{Sensitivity analysis}

The goal of mathematical modeling of infectious diseases is to provide insight into the epidemiology of the disease in the population. According to [45], it can be used to understand how infectious agents such as viruses, or bacteria spread in a population. Hence, the model results need to be able to provide insight into the dynamics of the disease. One of the techniques in providing such valuable insight is sensitivity analysis (SA). In this section, we carried out a SA to assess the relationship between the model parameters. This will inform us of the impact of each parameter on the threshold quantity (reproduction number), and hence enlighten the public health and policymakers to put priority on the intervention strategy for preventing and controlling the spread of the disease. Using the approach in [46, 47], the normalized forward sensitivity index $Z_{p}^{\mathcal{R}_{0}}$ on the reproduction number $\mathcal{R}_{0}$ for each of the parameters $p$, is defined as

$$
Z_{p}^{\mathcal{R}_{0}}=\frac{\partial \mathcal{R}_{0}}{\partial p} \times \frac{p}{\mathcal{R}_{0}}
$$

Employing the formula given by (21), with the parameter values provided in Table 2, the respective sensitivity indices values are presented in Table 3. We present a bar plot in Fig. 5 to further represent the numerical result of the sensitivity indices. It must be noted that an increase in any positive index from SA will directly increase the threshold quantity of the disease and vice versa, while an increase in the negative index will decrease the threshold quantity and vice versa. From Table 3 , increase in the spread of Lassa fever is associated to an increase in the positive values of the parameters, $\mu_{h}, \beta_{r}, \beta_{r h}, \beta_{h r}, \beta_{h}$, and $\pi_{r}$. Notable among the positive values are the transmission probabilities and the recruitment rate of rodents. In addition, increase in the spread of Lassa fever is associated to the decrease in the negative values of the parameters, $\pi_{h}, \delta_{h}, \tau_{h}$, and $\mu_{r}$. The natural death rate of rodents is noted as the highest negative value of the sensitivity index. The 


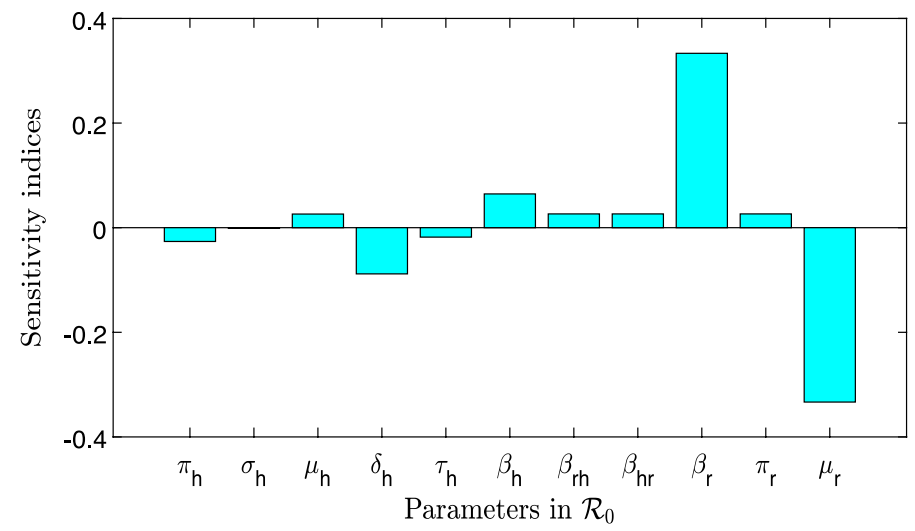

Fig. 5 Sensitivity indicies of the Lassa fever reproduction number $\mathcal{R}_{0} 8$

Table 3 Normalized sensitivity index of the reproduction number (9) parameters

\begin{tabular}{llll}
\hline Parameter & Description & Sensitivity Index & Sign \\
\hline$\pi_{h}$ & Recruitment rate of humans through birth or immigration & -0.0263 & - ve \\
$\sigma_{h}$ & Disease progression rate from exposed to infectious human & -0.0011 & - ve \\
$\mu_{h}$ & Natural death rate of humans & +0.0261 & + ve \\
$\delta_{h}$ & Disease induced death rate for humans & -0.0883 & - ve \\
$\tau_{h}$ & Recovery rate of infectious humans & -0.0180 & - ve \\
$\beta_{h}$ & Transmission probability from human-to-human & +0.0645 & + ve \\
$\beta_{r h}$ & Transmission probability from rodent-to-human & +0.0263 & + ve \\
$\beta_{h r}$ & Transmission probability from human-to-rodent & +0.0263 & + ve \\
$\beta_{r}$ & Transmission probability from rodent-to-rodent & +0.3333 & + ve \\
$\pi_{r}$ & Recruitment rate of rodents through birth & +0.0263 & + ve \\
$\mu_{r}$ & Natural death rate of rodents & -0.3333 & - ve \\
$\mathcal{R}_{0}$ & Reproduction number & 1.32 & \\
\hline
\end{tabular}

results enlighten us of the control strategies that are suitable in mitigating the spread of Lassa fever in the population. For example, the positive index +0.3333 of the transmission probability from rodent-to-rodent $\beta_{r}$ implies that increase (or decrease) by $1 \%$ of the value of $\beta_{r}$ will cause a corresponding increase (or decrease) in the reproduction number by $1 \%$. Also, the negative index -0.3333 of the natural death of rodents $\mu_{r}$ implies that an increase (or decrease) by $1 \%$ of the value of $\mu_{r}$ will cause a corresponding decrease (or increase) in the reproduction number by $1 \%$.

In summary, the Lassa fever sensitivity analysis carried out suggests that any control strategies that reduce the transmission probabilities and the recruitment rate of rodents in the population will effectively curtail the spread of Lassa fever in the populace. An example of such a control mechanism is promoting good environmental and personal hygiene, which can be encouraged through educational campaigns, to avoid contamination of human foods by rodents. In addition, any control strategies that increase the death of rodents, such as the use of rodent traps or pesticides for fumigating the environment, will help in reducing the spread of Lassa fever. 


\section{Results and discussion}

To establish our theoretical findings, we present the numerical simulation results of the model in this section. Following the result from the sensitivity analysis, we investigate the effect of the most sensitive parameters on the reproduction number. In addition, we examine the dynamical behavior of infected human and rodent populations under different scenarios to predict the eradication of Lassa fever in Nigeria. It must be noted that, since Lassa fever exposed humans can transmit the infection, we defined the total infected human population as the sum of both exposed human and infectious human $\left(E_{h}+I_{h}\right)$. We developed a program code written and implemented on MATLAB ODE45 solvers, a six-stage fifth-order Runge-Kutta method, to simulate the model system (1). All the parameter values used are provided in Table 2, except otherwise stated. These values were obtained by fitting the real data reported by NCDC to the model (1), as presented in Section. Since these real data are reported cases specifically from Nigeria, the prediction of the numerical simulation results will be suitable for the description of the transmission dynamics of Lassa fever in Nigeria. The selection of our initial conditions is based on the reported real data and the demographic data of Nigeria. We assume the initial exposed human population as the first reported case of Lassa fever given as $E_{h}(0)=98$; the initial infectious human population is assumed to be the first confirmed case of Lassa fever given as $I_{h}(0)=18$; and the initial recovered human population is assumed as $R_{h}(0)=0$. Since the total human population of Nigeria is reported as $N_{h}(0)=214,028,302$, thus we estimate the initial susceptible population as $S_{h}(0)=N_{h}(0)-\left(E_{h}(0)+I_{h}(0)+R_{h}(0)\right)$.

Since the reproduction number is the threshold quantity that determines the control or spread of disease in the population (except for cases where the bifurcation phenomenon occurs), we investigate the effect of some parameters (based on the results from the sensitivity analysis), on the reproduction number $\mathcal{R}_{0}$ in Fig. 6 . The effect of the transmission probability from rodent-to-rodent $\beta_{r}$ on the reproduction number is presented in Fig. 6a. It is obvious from the figure that an increase in the transmission probability from rodent-to-rodent directly increases the reproduction number. Similarly, as presented in Fig. $6 \mathrm{c}$, an increase in the transmission probability from human-to-human $\beta_{h}$ increases the reproduction number of the disease. These results are expected since the transmission of the infection increases the spread of Lassa fever in a population. Thus, an upsurge in the abundance of infected rodents or humans will result in an increase in the spread of Lassa fever in the population where prevention or control measures are not effective in use. Hence, an effort towards the reduction of disease transmission probabilities such as $\beta_{h}$ and $\beta_{r}$, will reduce the spread of Lassa fever in the population.

The effect of the natural death of rodents on the reproduction number is presented in Fig. 6b. An increase in the natural death of rodents reduces the reproduction number. However, it must be noted that after the fixed point $\mu_{r}=0.01$, the reproduction number remains stable regardless of a further increase in the death of rodents. This dynamic invalidates the expectation that continuous reduction of infected rodents should continually reduce the reproduction number. However, since a decrease in the $\mathcal{R}_{0}$ is not dependent on only the death of rodents, a combination of multiple control mechanisms can help to further reduce the reproduction number of the disease. A more interesting result is that of the effect of the recovery rate of human $\tau_{h}$ on the 


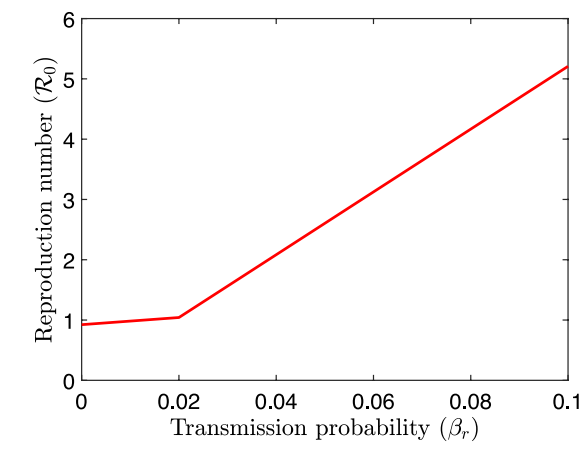

(a)

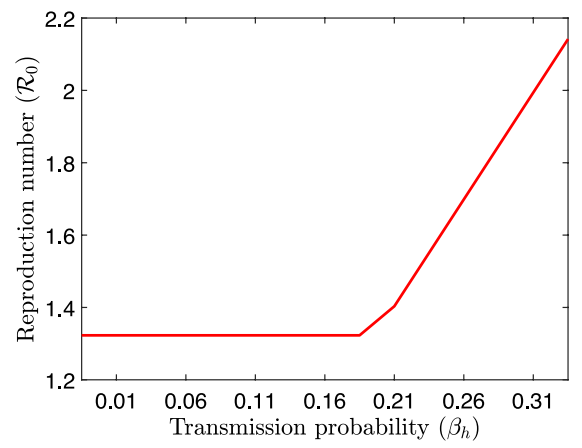

(c)

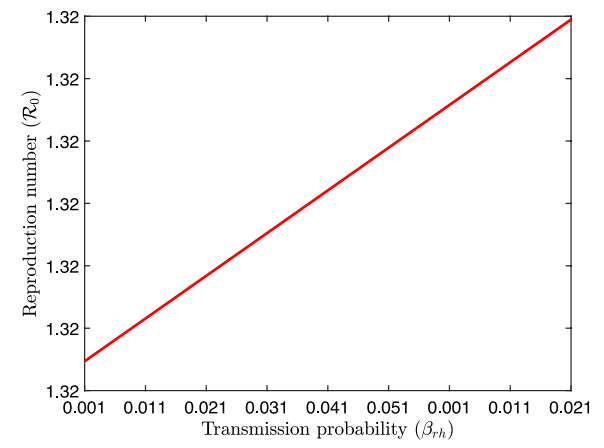

(e)

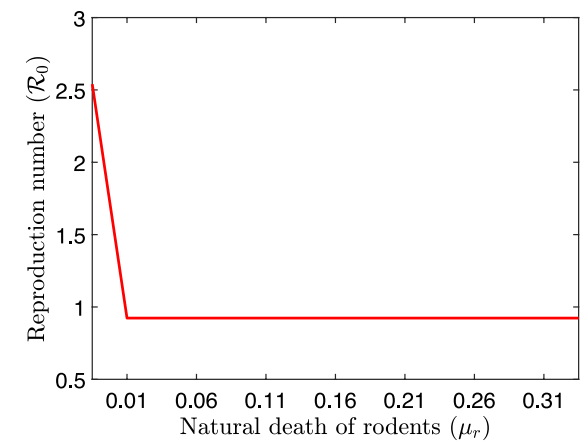

(b)

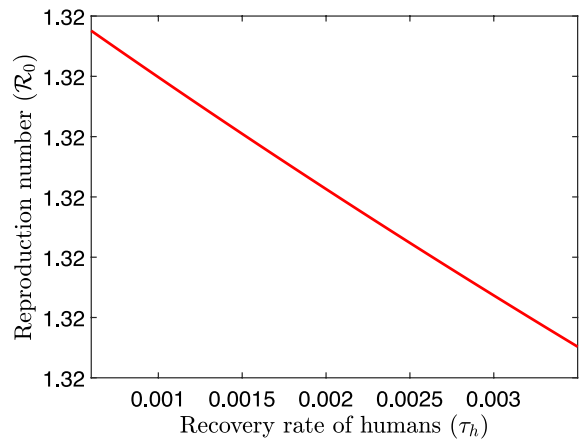

(d)

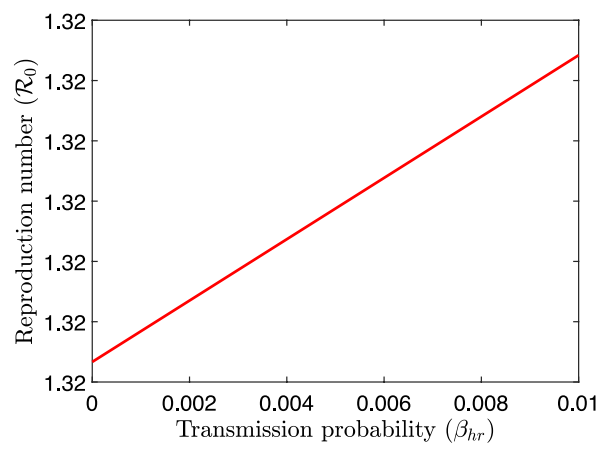

(f)

Fig. 6 Reproduction number $\mathcal{R}_{0}$ of Lassa fever model (1), with respect to model parameters. Parameter values used are as given in Table 2

reproduction number depicted in Fig. 6 d. An increase in the recovery rate of infected humans insignificantly decreases the reproduction number. The recovery rate of a human is as a function of increase in treatment of infected individuals, thus it is expected to see such insignificant decrease in the reproduction number, as treatment without any control measure that accounts for prevention of the disease will insignificantly or not reduce the burden of the disease, especially in a scenario where there are possibilities for loss of immunity as presented in our model.

Figure 6e, $\mathrm{f}$ respectively depict the effect of transmission probability from rodentto-human, and human-to-rodent on the reproduction number. An increase in the respective transmission probabilities increases the reproduction number. However, 


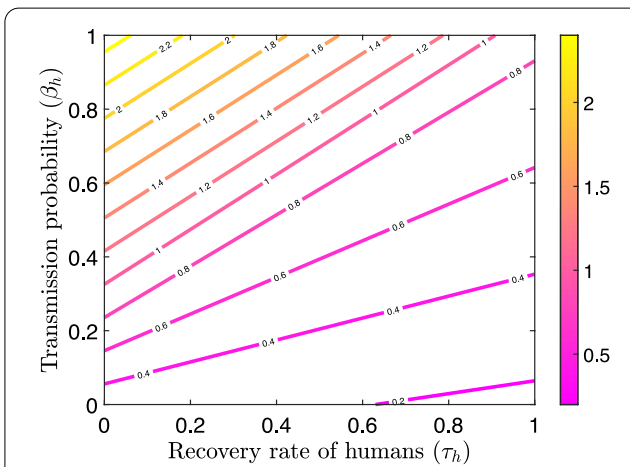

(a)

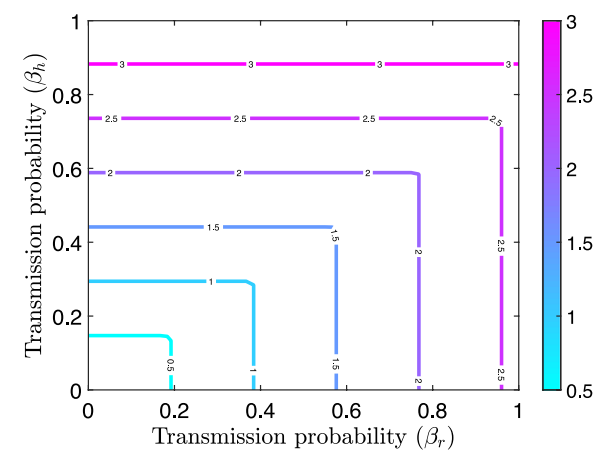

(c)

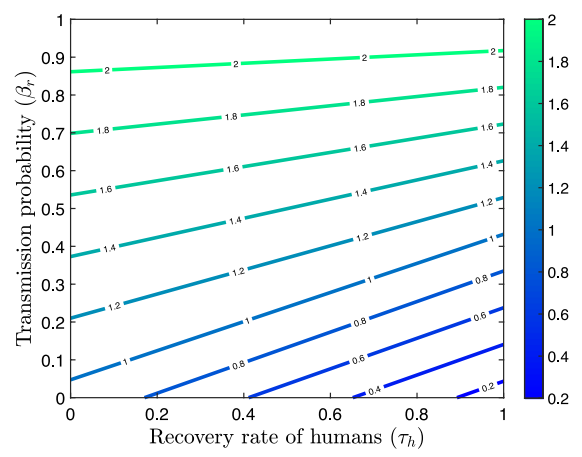

(b)

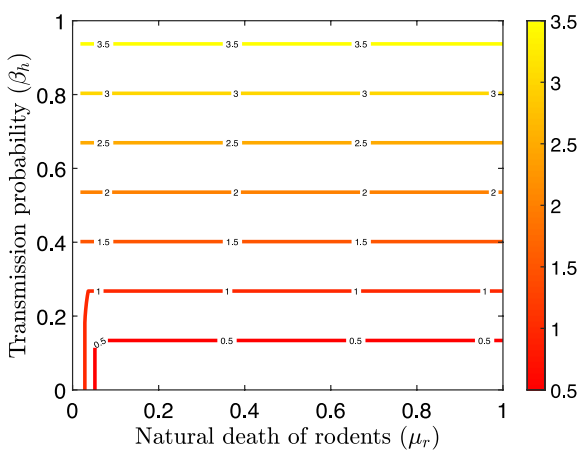

(d)

Fig. 7 2-D Contour plot of the reproduction number $\mathcal{R}_{0}$ of Lassa fever model (1), a varying recovery rate of humans with respect to transmission probability from human-to-human. $\mathbf{b}$ varying recovery rate of humans with respect to transmission probability from rodent-to-rodent. $\mathbf{c}$ transmission probability from rodent-to-rodent with respect to transmission probability from human-to-human. $\mathbf{d}$ natural death of rodents with respect to transmission probability from rodent-to-rodent. The parameter values used are as given in Table 2 except for $\delta_{h}=0.2911$ and $\mu_{r}=0.3840$, so that $\mathcal{R}_{0}=0.43<1$

the changes in the reproduction number estimate are very insignificant. Although this is not expected because an increase in the transmission of infection is expected to increase the disease burden in the population. Thus, we employ a 2-D contour plot to further illustrate the dynamics of the reproduction number, by varying two parameters simultaneously in Fig. 7. In Fig. 7a, we demonstrate the dynamics of the reproduction number by varying the recovery rate of human $\tau_{h}$, with respect to the transmission probability from human-to-human $\beta_{h}$. The result shows that simultaneous decrease of the transmission probability from human-to-human below 0.4 and continuous increase in the recovery rate of infected humans will keep the reproduction number below unity. A similar result is presented in Fig. 7b. The figure depicts the effect of varying the recovery rate of humans with respect to the transmission probability from rodent-to-rodent on the reproduction number. Keeping $\beta_{r}$ below 0.1 and simultaneously increasing the recovery rate of humans will alleviate the reproduction number below unity. Thus, it can be suggested that to stabilize the $\mathcal{R}_{0}$ below unity, a control strategy that reduces the transmission of Lassa fever between humans $\beta_{h}$, and rodents $\beta_{r}$, with control measure that enables an increase in recovery rate of infected humans should be sufficient to curtail the disease. The outcome of the transmission probability from human-to-human $\beta_{h}$ with respect to the transmission 
probability from rodent-to-rodent $\beta_{r}$ on the reproduction number is presented in Fig. 7c. An increase in any of the two parameters leads to an increase in $\mathcal{R}_{0}$. For instance, increasing $\beta_{r}$ while we fix $\beta_{h}=0$ leads to an increase in the reproduction number. Likewise, increasing $\beta_{h}$ while we fix $\beta_{r}=0$ leads to an increase in the reproduction number. To maintain the reproduction number of Lassa fever below unity, the values of the transmission probability from human-to-human and the transmission probability from rodent-to-rodent must be concurrently reduced below $\left(\beta_{h}<0.3, \beta_{r}<0.4\right)$. Hence, this result recommends that to decrease the reproduction number of Lassa fever below unity, it is not enough to only reduce one of the transmission probabilities, but any control strategies that facilitate the reduction in the transmission probability from human-to-human together with the transmission probability from rodent-to-rodent will help in reducing $\mathcal{R}_{0}$, thus leading to a reduction in the spread of Lassa fever in the populace. Figure $7 \mathrm{~d}$ depicts the effect of the transmission probability from human-to-human $\beta_{h}$ with respect to the natural death of rodents on the reproduction number. The result shows that increase in $\beta_{h}$ increases $\mathcal{R}_{0}$, while an increase in the death of rodents has no impact on the reproduction number. This correspond to the result from Fig. 6b (see discussion on Fig. 6b).

As stated in "Section Bifurcation analysis", the BB phenomenon suggests that the epidemiological condition of having the reproduction number less than unity to eliminate a disease although necessary is no longer enough for the effective control of the disease in the population. Hence, even though some parameters have no significant effect on the reproduction number as shown in Fig. 6b, d, e, f, it is important to further investigate the impact of parameters on the population, rather than on the reproduction number, since the model considered here exhibit the possibilities of bifurcation phenomenon.

It is important to mention that in Fig. 8, we simulate the effect of the most sensitive parameters (as suggested from the SA result), on the total infected human population. We aim to use the results from this simulation to predict and make recommendations for effective control measures that can facilitate the eradication of Lassa fever in Nigeria. To achieve this, we regulate (henceforth referred to as "control") the baseline parameter values by reducing the transmission probabilities $\beta_{h}, \beta_{r}, \beta_{h r}$ and $\beta_{r h}$ by $50 \%$ such that, $\beta_{h}=0.063, \beta_{r}=0.013, \beta_{h r}=0.007$ and $\beta_{r h}=0.026$. In addition, we increase the recovery rate of human and natural death of rodents by $50 \%$ such that, $\tau_{h}=0.005$, and $\mu_{r}=0.038$. Thus, we use the controlled parameters to simulate the dynamics of Lassa fever on the total infected human population. We depict the effect of each controlled parameter and the combination of different controlled parameters on the total infected human population in Fig. 8. Figure 8a illustrate the effect of $\beta_{r},\left(\beta_{r}\right.$ and $\left.\mu_{r}\right)$ and $\left(\beta_{r}\right.$, $\mu_{r}$, and $\beta_{h}$ ), on the infected human population. The result shows that using the three controlled parameters, the total infected human population declined faster compared to the effect of a single or double controlled parameter. Thus, simultaneous reduction of the transmission of Lassa fever from rodent-to-rodent $\beta_{r}$, the transmission of Lassa fever from human-to-human $\beta_{h}$, and increase in the death of rodents $\mu_{r}$, will decrease the burden of Lassa fever in the population. A similar result are presented in Fig. 8bf. In general, the results show that combined controlled parameters decrease the total infected human population quicker than using a single controlled parameter. 


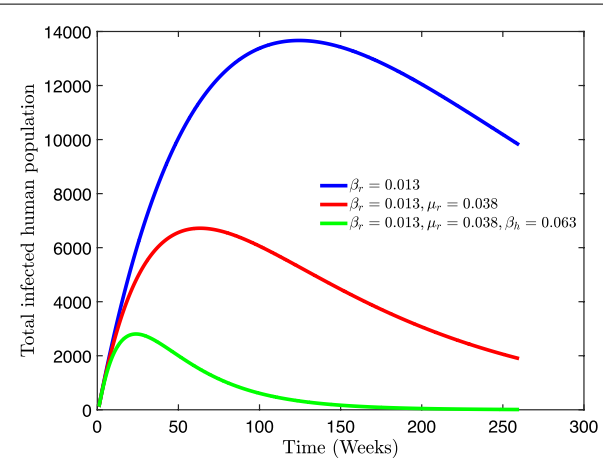

(a)

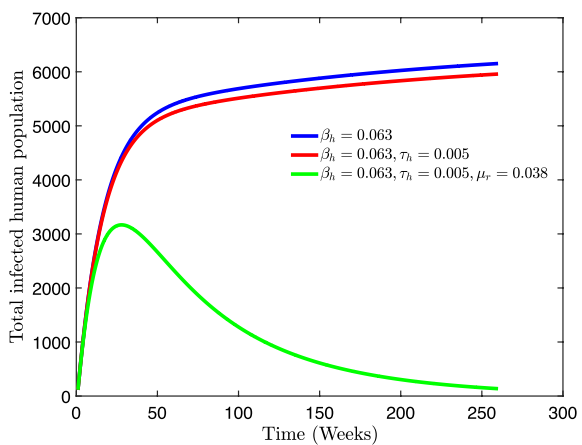

(c)

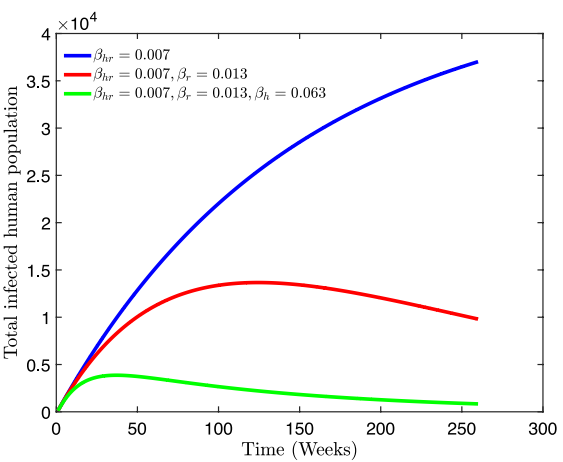

(e)

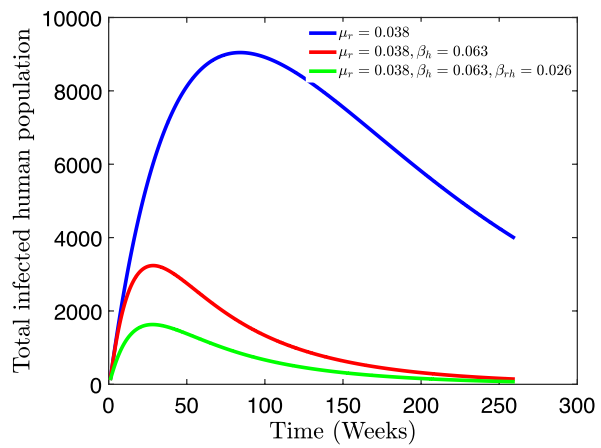

(b)

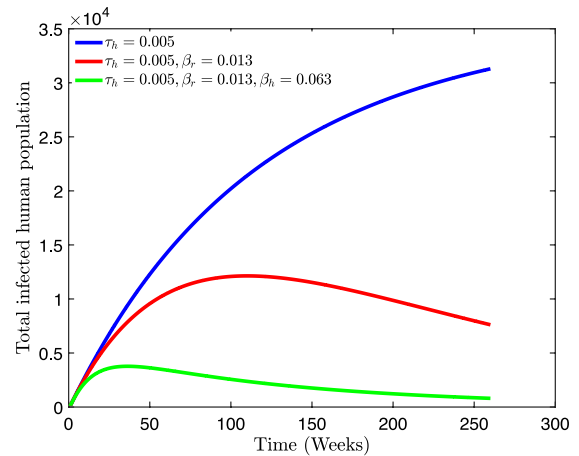

(d)

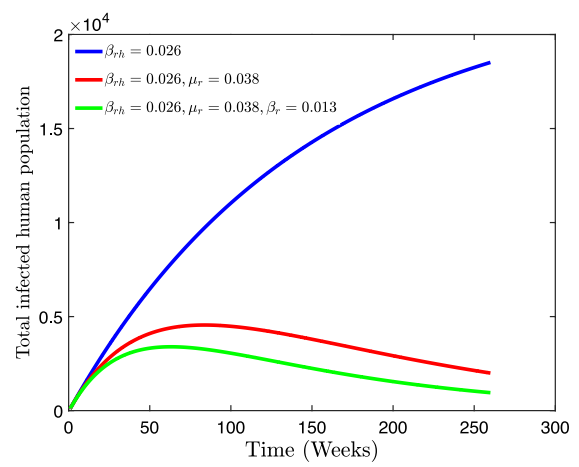

(f)

Fig. 8 Simulations of the Lassa fever model (1) showing the effects of controlled parameters on the total infected human population $\left(E_{h}+I_{h}\right)$. The parameter values used are as given in Table 2 except for $\beta_{h}=0.063, \beta_{r}=0.013, \beta_{h r}=0.007, \beta_{r h}=0.026, \tau_{h}=0.005$, and $\mu_{r}=0.038$

Following the result presented in Fig. 8, we present a simulation for the dynamics of the total infected human population under two different scenarios, in Fig. 9. The first scenario is with the baseline value of the parameters, characterized as "Nocontrols", while the second scenario is the combination of all controlled parameters based on the result from Fig. 8. Using the baseline parameter values, it is obvious that Lassa fever will persist in the population due to an increase in the infected human individuals. This is expected from the value of the reproduction number $\left(\mathcal{R}_{0}=1.32>1\right)$ according to Theorem 1 . On the other hand, combining all controlled parameters, the result shows that the existence of Lassa fever in the population extremely declined. The value of the reproduction number, using the value of 


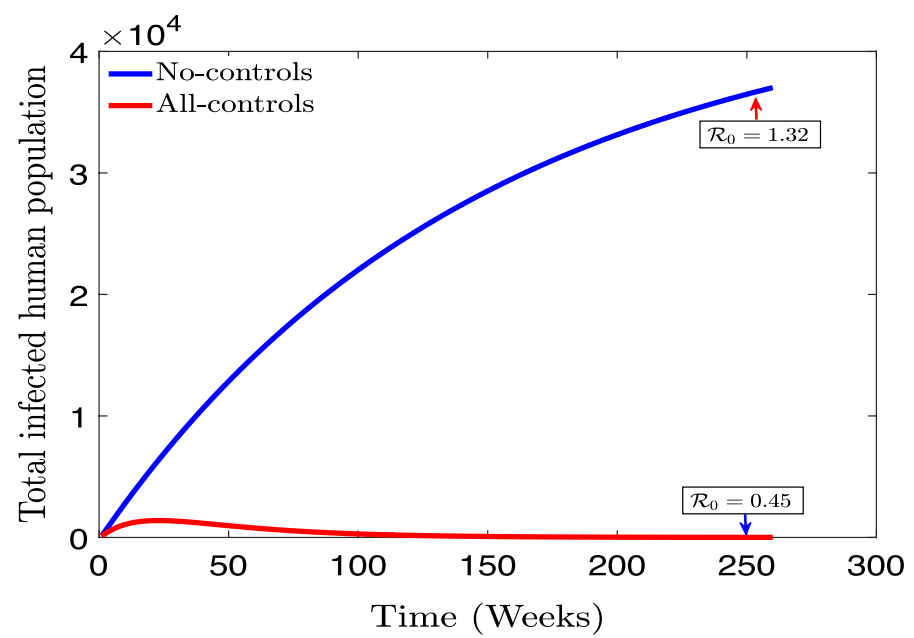

Fig. 9 Simulation of the dynamic of Lassa fever model (1), using parameter values as given in Table 2 such that $\mathcal{R}_{0}=1.32>1$ (No-controls) and parameter values as given in Table 2, except for $\beta_{h}=0.063, \beta_{r}=0.013, \beta_{h r}=0.007, \beta_{r h}=0.026, \tau_{h}=0.005$, and $\mu_{r}=0.038$ such that $\mathcal{R}_{0}=0.45<1$ (All-controls)

the controlled parameter is estimated as $\left(\mathcal{R}_{0}=0.45<1\right)$. Epidemiologically, the disease can be controlled in the population if the reproduction number of the disease is below unity. Thus, Lassa fever can be eradicated in Nigeria if there is an increase in efforts towards effective control measures that reduce the reproduction number of Lassa fever in Nigeria. Since the combination of all the controlled parameters reduces the burden of Lassa fever in the population (such that $\mathcal{R}_{0}=0.45<1$ ), we recommend control strategies that best describe the effect of these parameters. For example, $\beta_{h}, \beta_{r}, \beta_{h r}$ and $\beta_{r h}$ are transmission probabilities, thus any control strategy that will curtail the transmission of the disease such as; an educational campaign to enlighten the population about personal hygiene and also precaution by health practitioners taking care of infected patients; the use of a condom to prevent secondary transmission from human-to-human, will help in reducing the transmission of Lassa fever in Nigeria. In addition, for the controlled parameters $\mu_{r}$ and $\tau_{h}$, any control strategy that increases the death of rodents such as the use of pesticides, rodent traps and early treatment of infected individuals will help in reducing the burden of Lassa fever in Nigeria.

To investigate the stability behavior of the total infected human and rodent population, we use the different initial sizes of the population to depict the convergence of solution trajectories in Fig. 10. This validates the global stability result of Theorem 2. Figure 10a, b illustrate the convergence to the Lassa fever-free equilibrium irrespective of the initial sizes of the infected human and rodent in the population, while Fig. 10c, d illustrate the convergence to the Lassa fever endemic equilibrium regardless of the initial sizes of the infected human and rodent population. This result implies that, regardless of any perturbation or change in the initial size of the population, the infected human and rodent population equilibrium will remain the same. 


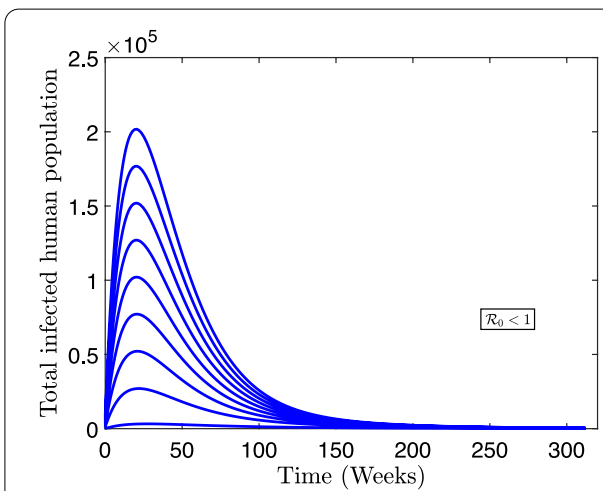

(a)

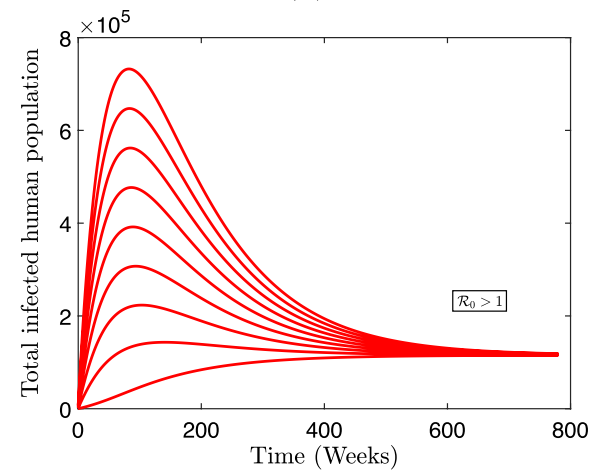

(c)

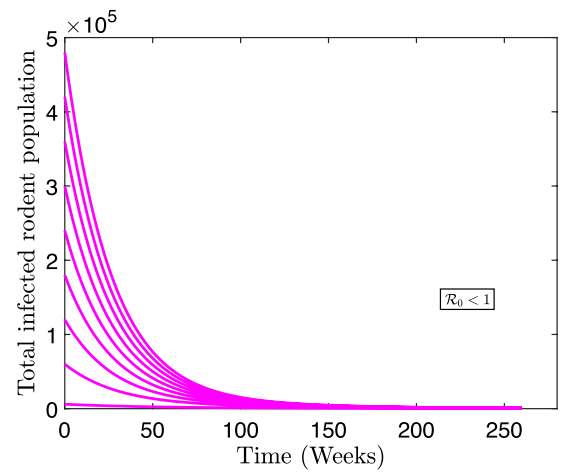

(b)

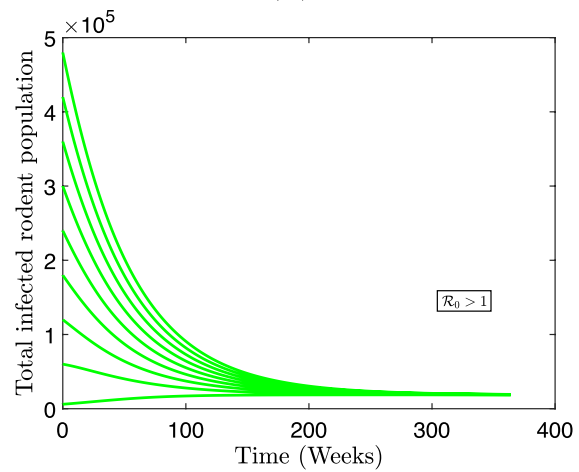

(d)

Fig. 10 Convergence of solution trajectories for total infected humans and rodents. $\mathbf{a}, \mathbf{b}$ The parameter values as given in Table 2 except for $\beta_{h}=0.063$, and $\mu_{r}=0.038$ such that $\mathcal{R}_{0}=0.67<1$. c, d The parameter values as given in Table 2 except for $\beta_{r}=0.051$, such that $\mathcal{R}_{0}=2.65>1$

\section{Conclusions}

In this study, we developed, analyzed, and simulated a deterministic model to describe the transmission dynamics of Lassa fever in Nigeria. Transmission of Lassa fever requires interaction between two-interacting hosts (namely human and rodent population), thus we sub-divided the human population into susceptible, exposed, infectious, and recovered humans, while the rodent population was subdivided into a susceptible and infectious rodents. We showed that the model is mathematically and epidemiologically meaningful by investigating the invariant region, the positivity of solutions, and boundedness. The local and global stability of the model was investigated using the reproduction number which was obtained by using the nextgeneration matrix. The result shows that the Lassa fever-free equilibrium $\mathcal{E}_{0}$ is locally and globally asymptotically stable if $\mathcal{R}_{0}<1$ and unstable otherwise. Furthermore, the endemic state of Lassa fever $\mathcal{E}_{1}$ exists for $\mathcal{R}_{0}>1$.

To best describe the dynamics of Lassa fever in Nigeria, we parameterized the formulated model (1) by using the cumulative reported cases data obtained from the Nigeria Centre for Disease and Control database. Reported cases used are from the first week of January 2020 through the eleventh week in 2021. Using these parameters obtained, we carried out a sensitivity analysis of the model parameters on the reproduction number to determine the impact of each parameter on the transmission 
of Lassa fever in Nigeria. Overall, the result shows that increase in the transmission of Lassa fever is associated to an increase in the transmission probabilities $\left(\beta_{h}, \beta_{r}, \beta_{h r}, \beta_{r h}\right)$ and increase in the number of rodents $\pi_{r}$ in the population. In addition, an increase in the death of rodents is associated with a decrease in the transmission of Lassa fever. Numerical simulations were carried out with parameterized data to describe the dynamics of Lassa fever in the population. We explored the effect of controlled parameters on the total infected human population. Results show that combined controlled parameters reduce the burden of Lassa fever faster in the population. Based on the result of the controlled parameters, we recommend control strategies that best describe the effects of these parameters. For example, $\beta_{h}, \beta_{r}, \beta_{h r}$ and $\beta_{r h}$ are transmission probabilities, thus any control strategy that will limit the transmission of the disease such as; an educational campaign to enlighten the population about personal hygiene and also precaution by health practitioners taking care of infected patients; the use of a condom to prevent secondary transmission from human-to-human, will help in reducing the transmission of Lassa fever in Nigeria. In addition, for the controlled parameters $\mu_{r}$ and $\tau_{h}$, any control strategy that increases the death of rodents such as the use of pesticides, rodent traps and early treatment of infected individuals will help in reducing the burden of Lassa fever in Nigeria.

Conclusively, to mitigate the burden of Lassa fever in each region of Africa where it is endemic, it will be beneficial to investigate the impact of using multiple control strategies in eradicating the disease. For the future direction of this study, we shall extend the model considered here by including the optimal control problem, using Pontryagin's maximum principle. Since eliminating any form of the disease in a large and underdeveloped population can be severe and expensive, we will investigate the most cost-effective strategy appropriate to use among several combinations of control measures using costeffectiveness analysis.

\section{Appendix}

Proof of Theorem 1

Proof To prove the Theorem 1, we obtain the Jacobian matrix by evaluating the model (1) at Lassa fever-free equilibrium $\mathcal{E}_{0}$ as

$$
\mathcal{J}\left(\mathcal{E}_{0}\right)=\left(\begin{array}{cccccc}
-\mu_{h} & 0 & -\beta_{h} & \xi_{h} & 0 & -\beta_{r h} \\
0 & -k_{1} & \beta_{h} & 0 & 0 & \beta_{r h} \\
0 & \sigma_{h} & -k_{2} & 0 & 0 & 0 \\
0 & 0 & \tau_{h} & -k_{3} & 0 & 0 \\
0 & 0 & -\frac{S_{r}^{*} \beta_{h r}}{S_{h}^{*}} & 0 & -\mu_{r} & -\beta_{r} \\
0 & 0 & \frac{S_{r}^{*} \beta_{h r}}{S_{h}^{*}} & 0 & 0 & -\mu_{r}+\beta_{r}
\end{array}\right)
$$

where: $k_{1}=\sigma_{h}+\mu_{h}, k_{2}=\tau_{h}+\mu_{h}+\delta_{h}$, and $k_{3}=\mu_{h}+\xi_{h}$. From (22), it is sufficient to show that all the eigenvalues of $\mathcal{J}\left(\mathcal{E}_{0}\right)$ are negative. We obtain the first three eigenvalues 
as, $-\mu_{r},-\mu_{h}$, and $-k_{3}$. The remaining eigenvalues can be obtained from the sub-matrix $\mathcal{J}_{1}\left(\mathcal{E}_{0}\right)$, which is written as

$$
\mathcal{J}_{1}\left(\mathcal{E}_{0}\right)=\left(\begin{array}{ccc}
-k_{1} & \beta_{h} & \beta_{r h} \\
\sigma_{h} & -k_{2} & 0 \\
0 & \frac{S_{r}^{*} \beta_{h r}}{S_{h}^{*}} & -\left(\mu_{r}-\beta_{r}\right)
\end{array}\right)
$$

The remaining three eigenvalues with negative real parts can be obtained through the characteristics polynomial of $\mathcal{J}_{1}\left(\mathcal{E}_{0}\right)$, given as

$$
\omega_{1} \lambda^{3}+\omega_{2} \lambda^{2}+\omega_{3} \lambda+\omega_{4}=0
$$

where

$$
\begin{aligned}
& \omega_{1}=1 \\
& \omega_{2}=\left(k_{1}+k_{2}\right)+\mu_{r}\left(1-\mathcal{R}_{r}\right) \\
& \omega_{3}=\mu_{r}\left(k_{1}+k_{2}\right)\left(1-\mathcal{R}_{r}\right)+k_{1} k_{2}\left(1-\mathcal{R}_{h}\right) \\
& \omega_{4}=\mu_{r} k_{1} k_{2}\left(1-\mathcal{R}_{r}\right)\left(1-\mathcal{R}_{h}\right)\left\{1-\frac{\mathcal{R}_{h r} \mathcal{R}_{r h}}{\left(1-\mathcal{R}_{h}\right)\left(1-\mathcal{R}_{r}\right)}\right\}
\end{aligned}
$$

It is obvious that the coefficient $\omega_{1}$ is positive, while $\omega_{i}$ for $i=2, \ldots, 4$ can be positive or negative depending on the values of respective reproduction number $\mathcal{R}_{h}$ and $\mathcal{R}_{r}$. For the disease free equilibrium case, the condition $\mathcal{R}_{h}<1$ and $\mathcal{R}_{r}<1$ must be satisfied, so that the coefficient $\omega_{i}$ for $i=2, \ldots, 4$ will be positive. In addition, for the coefficient $\omega_{4}$ to be positive, the condition $\frac{\mathcal{R}_{h h} \mathcal{R}_{r h}}{\left(1-\mathcal{R}_{h}\right)\left(1-\mathcal{R}_{r}\right)}<1$ must be satisfied.

Now, applying the Routh-Hurwitz stability criterion for the third-order polynomial [48, 49], equation (24) will have roots with negative real parts if and only if the coefficients $\omega_{i}$ are positive for $i=2, \ldots, 4$ and $\omega_{2} \omega_{3}>\omega_{4}$. Hence, the conditions of the Routh-Hurwitz criterion established the local asymptomatic stability of the Lassa fever model given by (1) at the disease-free equilibrium $\mathcal{E}_{0}$.

\section{Proof of Theorem 2}

Proof From the Lassa fever model (1), we can obtain $F(X, Z)$, and $G(X, Z)$ as

$$
\frac{\mathrm{d} X}{\mathrm{~d} t}=F(X, Z)=\left(\begin{array}{c}
\pi_{h}+\xi_{h} R_{h}-\lambda_{h} S_{h}-\mu_{h} S_{h} \\
\tau_{h} I_{h}-k_{3} R_{h} \\
\pi_{r}-\lambda_{r} S_{r}-\mu_{r} S_{r}
\end{array}\right), \quad \frac{\mathrm{d} Z}{\mathrm{~d} t}=G(X, Z)=\left(\begin{array}{c}
\lambda_{h} S_{h}-k_{1} E_{h} \\
\sigma_{h} E_{h}-k_{2} I_{h} \\
\lambda_{r} S_{r}-\mu_{r} I_{r}
\end{array}\right)
$$

where $k_{1}=\left(\sigma_{h}+\mu_{h}\right), k_{2}=\left(\tau_{h}+\mu_{h}+\delta_{h}\right)$, and $k_{3}=\left(\mu_{h}+\xi_{h}\right)$. From (25), we obtain the reduced system below: 


$$
\left.\frac{\mathrm{d} X}{\mathrm{~d} t}\right|_{Z=0}=F(X, 0)=\left(\begin{array}{c}
\pi_{h}-\mu_{h} S_{h} \\
0 \\
\pi_{r}-\mu_{r} S_{r}
\end{array}\right)
$$

From equation (26), it is obvious that $\mathcal{E}_{0}^{*}=\left(\frac{\pi_{h}}{\mu_{h}}, 0, \frac{\pi_{r}}{\mu_{r}}\right)$ is the GAS equilibrium point for the reduced system (26). This is trivia by solving $\frac{\mathrm{d} S_{h}}{\mathrm{~d} t}=\pi_{h}-\mu_{h} S_{h}$ to obtain $S_{h}(t)=\frac{\pi_{h}}{\mu_{h}}+\left(S_{h}(0)-\frac{\pi_{h}}{\mu_{h}}\right) \exp ^{-\mu_{h} t}$, which implies that $S_{h} \rightarrow \frac{\pi_{h}}{\mu_{h}}$ as $t \rightarrow \infty$. Similarly, it can be shown that $S_{r} \rightarrow \frac{\pi_{r}}{\mu_{r}}$ as $t \rightarrow \infty$. Hence, the convergence of solutions is global in the region $\mathcal{D}$. Let,

$$
Q=B_{Z} G\left(X^{*}, 0\right)=\left(\begin{array}{ccc}
-k_{1} & \beta_{h} & \beta_{r h} \\
\sigma_{h} & -k_{2} & 0 \\
0 & \frac{S_{r}^{*} \beta_{h r}}{S_{h}^{*}} & -\left(\mu_{r}-\beta_{r}\right)
\end{array}\right)
$$

Then, we verify the second condition $(C 2)$ :

$$
\hat{G}(X, Z)=\left(\begin{array}{l}
\hat{G}_{1}(X, Z) \\
\hat{G}_{2}(X, Z) \\
\hat{G}_{3}(X, Z)
\end{array}\right)=\left(\begin{array}{c}
\left(\beta_{h} I_{h}+\beta_{r h} I_{r}\right)\left(1-\frac{S_{h}}{N_{h}}\right) \\
0 \\
\frac{\beta_{h r} I_{h} S_{r}^{*}}{S_{h}^{*}}\left(1-\frac{S_{r} S_{h}^{*}}{S_{r}^{*} N_{h}}\right)+\beta_{r} I_{r}\left(1-\frac{S_{r}}{N_{r}}\right)
\end{array}\right)
$$

Hence, since $0 \leq S_{h}$ and $0 \leq S_{r}$, it is clear that $\hat{G}(X, Z) \geq 0$. Thus, the Lassa fever-free with the fixed point $\mathcal{E}_{0}^{*}=\left(X^{*}, 0\right)$ is globally asymptotically stable when $\mathcal{R}_{0}<1$.

\section{Coefficients of polynomial (14)}

$$
\begin{aligned}
a_{1} & =\beta_{r} \mu_{r}^{2}\left\{\pi_{h}\left(k_{2} k_{3}+k_{3} \sigma_{h}+\sigma_{h} \tau_{h}\right)\right\}^{3} \\
a_{2} & =J \mu_{r}\left(J Q \mu_{r} \phi_{3}+3 J \beta_{r} \mu_{r} \phi_{2}+Q \phi_{1} \phi_{3}\right)-J^{2} \beta_{r} \mu_{r}\left(Q \phi_{3}+2 \phi_{5}\right) \\
a_{3} & =J \mu_{r}\left(J \mu_{r} \phi_{3} \phi_{4}+2 Q \phi_{2} \phi_{3} \mu_{r}+3 \beta_{r} \mu_{r} \phi_{2}^{2}+\phi_{1} \phi_{3} \phi_{4}\right)+Q \phi_{3}\left(J \beta_{r} \phi_{5}+\mu_{r} \phi_{1} \phi_{2}\right)+J \beta_{r} \phi_{5}^{2} \\
& -\left\{J \mu_{r}\left(J \beta_{r} \phi_{3} \phi_{4}+2 Q \beta_{r} \phi_{2} \phi_{3}+Q \phi_{3} \phi_{5}+4 \beta_{r} \phi_{2} \phi_{5}\right)+Q \phi_{1} \phi_{3}\left(Q \phi_{3}+\phi_{5}\right)\right\} \\
a_{4} & =\mu_{r} \phi_{2}\left(2 J \mu_{r} \phi_{3} \phi_{4}+Q \mu_{r} \phi_{2} \phi_{3}+\beta_{r} \mu_{r} \phi_{2}^{2}+\phi_{1} \phi_{3} \phi_{4}\right)+\beta_{r} \phi_{2} \phi_{5}\left(Q \phi_{3}+\phi_{5}\right)+J \beta_{r} \phi_{3} \phi_{4} \phi_{5} \\
& -\left\{\mu_{r} \phi_{2}\left(2 J \phi_{3} \phi_{4} \beta_{r}+Q \beta_{r} \phi_{2} \phi_{3}+Q \phi_{3} \phi_{5}+2 \beta_{r} \phi_{2} \phi_{5}\right)+\phi_{3} \phi_{4}\left(J \mu_{r} \phi_{5}+2 Q \phi_{1} \phi_{3}+\phi_{1} \phi_{5}\right)\right\} \\
a_{5} & =\left(1-\mathcal{R}_{h}\right)+\mathcal{R}_{h} \mathcal{R}_{r}\left(1-\frac{1}{k_{1} k_{2} \mathcal{R}_{h}}\right)-\mathcal{R}_{h r} \mathcal{R}_{r h}
\end{aligned}
$$

where: $\phi_{1}=\beta_{h r} \pi_{h} \sigma_{h} k_{3}, \phi_{2}=\pi_{h} k_{1} k_{2} k_{3}, \phi_{3}=\beta_{r h} \pi_{r}, \phi_{4}=\mu_{h} k_{1} k_{2} k_{3}, \phi_{5}=\mu_{r} \beta_{h} \pi_{h} \sigma_{h} k_{3}$, $J=\pi_{h}\left(k_{2} k_{3}+k_{3} \sigma_{h}+\sigma_{h} \tau_{h}\right)$ and $Q=k_{1} k_{2} k_{3}-\sigma_{h} \tau_{h} \xi_{h}$. 


\section{Abbreviations}

LF: Lassa fever; NGM: Next generation matrix; SA: Sensitivity analysis; NCDC: Nigeria Centre for Disease Control; WHO: World Health Organization; CDC: Centers for Disease Control and Prevention; MATLAB: Matrix Laboratory.

\section{Acknowledgements}

Not applicable.

\section{Authors' contributions}

MMO developed and carried out the analysis of the model. MMO performed the model fitting and simulations, while EFDG supervised the project. All authors read and approved the final manuscript.

\section{Funding}

Not applicable

\section{Availability of data and materials}

All data supporting the findings of this study are included in the list of references and can be obtained at the Nigeria Centre for Disease Control (NCDC) http://www.ncdc.gov.ng/reports.

\section{Declarations}

\section{Competing interests}

The authors declare that they have no competing interests.

\section{Author details}

${ }^{1}$ Department of Mathematical Sciences, University of South Africa, Florida, South Africa. ${ }^{2}$ Thermo Fisher Scientific, Microbiology Division, Lenexa, USA.

Received: 26 July 2021 Accepted: 13 January 2022

Published online: 25 January 2022

\section{References}

1. Peterson, A.T., Moses, L.M., Bausch, D.G.: Mapping transmission risk of Lassa fever in West Africa: the importance of quality control, sampling bias, and error weighting. PLOS ONE 9(8), 100711 (2014)

2. Ibrahim, M.A., Dénes, A.: A mathematical model for Lassa fever transmission dynamics in a seasonal environment with a view to the 2017-20 epidemic in nigeria. Nonlinear Anal. Real World Appl. 60, 103310 (2021)

3. Zhao, S., Musa, S.S., Fu, H., He, D., Qin, J.: Large-scale Lassa fever outbreaks in Nigeria: quantifying the association between disease reproduction number and local rainfall. Epidemiol. Infect. 148, 10 (2020)

4. Gibb, R., Moses, L.M., Redding, D.W., Jones, K.E.: Understanding the cryptic nature of Lassa fever in West Africa. Pathogens Global Health 111(6), 276-288 (2017)

5. Richmond, J.K., Baglole, D.J.: Lassa fever: epidemiology, clinical features, and social consequences. BMJ 327(7426), 1271-1275 (2003)

6. Greenky, D., Knust, B., Dziuban, E.J.: What pediatricians should know about Lassa virus. JAMA Pediatr. 172(5), 407-408 (2018)

7. Mariën, J., Borremans, B., Kourouma, F., Baforday, J., Rieger, T., Günther, S., Magassouba, N., Leirs, H., Fichet-Calvet, E.: Evaluation of rodent control to fight Lassa fever based on field data and mathematical modelling. Emerg. Microbes Infect. 8(1), 640-649 (2019)

8. Olugasa, B.O., Odigie, E.A., Lawani, M., Ojo, J.F., et al.: Development of a time-trend model for analyzing and predicting case-pattern of Lassa fever epidemics in Liberia, 2013-2017. Ann. Afr. Med. 14(2), 89 (2015)

9. Musa, S.S., Zhao, S., Gao, D., Lin, Q., Chowell, G., He, D.: Mechanistic modelling of the large-scale Lassa fever epidemics in Nigeria from 2016 to 2019. J. Theor. Biol. 493, 110209 (2020)

10. Davies, J., Lokuge, K., Glass, K.: Routine and pulse vaccination for Lassa virus could reduce high levels of endemic disease: a mathematical modelling study. Vaccine 37(26), 3451-3456 (2019)

11. Peter, O.J., Abioye, A.I., Oguntolu, F.A., Owolabi, T.A., Ajisope, M.O., Zakari, A.G., Shaba, T.G.: Modelling and optimal control analysis of Lassa fever disease. Inform. Med. Unlocked 20, 100419 (2020)

12. Maxmen, A.: Deadly Lassa-fever outbreak tests Nigeria's revamped health agency. Nature 555, 7697 (2018)

13. Nigeria Centre for Disease Control:Weekly epidemiological report. Available from https://ncdc.gov.ng/reports/ weekly

14. Onah, I.S., Collins, O.C.: Dynamical system analysis of a Lassa fever model with varying socioeconomic classes. J. Appl. Math. 2020 (2020)

15. Hamblion, E., Raftery, P., Wendland, A., Dweh, E., Williams, G., George, R., Soro, L., Katawera, V., Clement, P., Gasasira, A., et al.: The challenges of detecting and responding to a Lassa fever outbreak in an Ebola-affected setting. Int. J. Infect. Dis. 66, 65-73 (2018)

16. Bakare, E., Are, E., Abolarin, O., Osanyinlusi, S., Ngwu, B., Ubaka, O.N.: Mathematical modelling and analysis of transmission dynamics of Lassa fever. J. Appl. Math. 2020 (2020)

17. Srivastava, H.M., AbuJarad, E.S., Jarad, F., Srivastava, G., AbuJarad, M.H.: The Marichev-Saigo-Maeda fractional-calculus operators involving the (p, q)-extended bessel and bessel-wright functions. Fractal Fract. 5(4), 210 (2021)

18. Srivastava, H.M., El-Sayed, A., Hashem, H., Al-Issa, S.M.: Analytical investigation of nonlinear hybrid implicit functional differential inclusions of arbitrary fractional orders. Revista de la Real Academia de Ciencias Exactas, Físicas y Naturales. Serie A. Matemáticas 116(1), 1-19 (2022) 
19. Srivastava, H.M.: Area Carracedo. Power-series solution of compartmental epidemiological models, I.C., Nieto Roig, J.J. (2021)

20. Singh, H., Srivastava, H.M., Hammouch, Z., Nisar, K.S.: Numerical simulation and stability analysis for the fractionalorder dynamics of Covid-19. Results Phys. 20, 103722 (2021)

21. Goufo, E.F.D.: A biomathematical view on the fractional dynamics of cellulose degradation. Fract. Calc. Appl. Anal. 18(3), 554-564 (2015)

22. Gbadamosi, B., Ojo, M.M., Oke, S.I., Matadi, M.B.: Qualitative analysis of a dengue fever model. Math. Comput. Appl. 23(3), 33 (2018)

23. Goufo, E.F.D., Pene, M.K., Mugisha, S.: Stability analysis of epidemic models of Ebola hemorrhagic fever with nonlinear transmission. J. Nonlinear Sci. Appl. (JNSA) 9(6), 4191-4205 (2016)

24. Akinpelu, F., Ojo, M.: A mathematical model for the dynamic spread of infection caused by poverty and prostitution in Nigeria. Int. J. Math. Phys. Sci. Res. 4, 33-47 (2016)

25. Doungmo Goufo, E.F., Oukouomi Noutchie, S.C., Mugisha, S.: A fractional seir epidemic model for spatial and temporal spread of measles in metapopulations. In: Abstract and Applied Analysis, vol. 2014 (2014). Hindawi

26. Akinpelu, F., Ojo, M.: Mathematical analysis of effect of isolation on the transmission of Ebola virus disease in a population. Asian Res. J. Math. 1, 1-12 (2016)

27. Castillo-Chavez, C., Song, B.: Dynamical models of tuberculosis and their applications. Math. Biosci. Eng. 1(2), 361 (2004)

28. Oke, S.I., Ojo, M.M., Adeniyi, M.O., Matadi, M.B.: Mathematical modeling of malaria disease with control strategy. Commun. Math. Biol. Neurosci. 2020 (2020)

29. Ojo, M.M.: Mathematical modeling of Neisseria meningitidis: a case study of Nigeria. Ph.D. thesis, University of Kansas (2019)

30. Singh, H.: Analysis for fractional dynamics of Ebola virus model. Chaos Solitons Fractals 138, 109992 (2020)

31. Srivastava, H.M., Saad, K.M., Khader, M.: An efficient spectral collocation method for the dynamic simulation of the fractional epidemiological model of the Ebola virus. Chaos Solitons Fractals 140, 110174 (2020)

32. Atangana, A., Goufo, E.F.D.: On the mathematical analysis of ebola hemorrhagic fever: deathly infection disease in West African countries. BioMed Res. Int. 2014 (2014)

33. Goufo, E.F.D., Atangana, A.: On analysis generalization of TB-HIV dynamics by a two-scale reduction process. Results Phys. 30, 104772 (2021)

34. Dachollom, S., Madubueze, C.E.: Mathematical model of the transmission dynamics of Lassa fever infection with controls. Math. Model Appl. 5, 65-86 (2020)

35. Fichet-Calvet, E., Rogers, D.J.: Risk maps of Lassa fever in West Africa. PLoS Negl. Trop. Dis. 3(3), 388 (2009)

36. Ojo, M.M., Gbadamosi, B., Benson, T.O., Adebimpe, O., Georgina, A.: Modeling the dynamics of Lassa fever in Nigeria. J. Egypt. Math. Soc. 29(1), 1-19 (2021)

37. Viral Hemorrhagic Fever Consortium: Diagnosis and symptoms of Lassa. Available from https://vhfc.org/diseases/ lassa/

38. Lakshmikantham, V., Leela, S., Martynyuk, A.A.: Stability Analysis of Nonlinear Systems. Springer, Berlin (1989)

39. Ojo, M., Akinpelu, F.: Lyapunov functions and global properties of seir epidemic model. Int. J. Chem. Math. Phys. 1(1), $1(2017)$

40. Diekmann, O., Heesterbeek, J.A.P., Metz, J.A.: On the definition and the computation of the basic reproduction ratio $r$ 0 in models for infectious diseases in heterogeneous populations. J. Math. Biol. 28(4), 365-382 (1990)

41. Diekmann, O., Heesterbeek, J., Roberts, M.G.: The construction of next-generation matrices for compartmental epidemic models. J. R. Soc. Interface 7(47), 873-885 (2010)

42. Castillo-Chavez, C., Blower, S., Van den Driessche, P., Kirschner, D., Yakubu, A.-A.: Mathematical Approaches for Emerging and Reemerging Infectious Diseases: Models, Methods, and Theory, vol. 126. Springer, Berlin (2002)

43. Central Intelligence Agency: The world factbook. Available from https://www.cia.gov/the-world-factbook/count ries/nigeria/

44. Nigeria Centre for Disease Control: Weekly epidemiological report. Available from https://ncdc.gov.ng/diseases/ info/L

45. Panovska-Griffiths, J.: Can Mathematical Modelling Solve the Current Covid-19 Crisis? Springer, Berlin (2020)

46. Ojo, M., Gbadamosi, B., Olukayode, A., Oluwaseun, O.R.: Sensitivity analysis of dengue model with saturated incidence rate. Open Access Library J. 5(03), 1 (2018)

47. Ojo, M., Akinpelu, F.: Sensitivity analysis of Ebola virus model. Asian Research J. Math. 1, 1-10 (2017)

48. Murray, J.: Mathematical Biology II: Spatial Models and Biomedical Applications, vol. 3. Springer, Berlin (2001)

49. Polyanin, A.D., Manzhirov, A.V.: Handbook of Mathematics for Engineers and Scientists. CRC Press, Cambridge (2006)

\section{Publisher's Note}

Springer Nature remains neutral with regard to jurisdictional claims in published maps and institutional affiliations. 